\title{
KEPLER-432: A RED GIANT INTERACTING WITH ONE OF ITS TWO LONG-PERIOD GIANT PLANETS
}

\author{
Samuel N. Quinn ${ }^{1,18}$, Timothy. R. White ${ }^{2}$, David W. Latham ${ }^{3}$, William J. Chaplin ${ }^{4,5}$, Rasmus Handberg ${ }^{4,5}$, \\ Daniel Huber ${ }^{6,7,5}$, David M. Kipping ${ }^{3,17}$, Matthew J. Payne ${ }^{3}$, Chen Jiang ${ }^{5}$, Victor Silva Aguirre ${ }^{5}$, Dennis Stello ${ }^{6,5}$, \\ David H. Sliski $^{3,8}$, David R. Ciardi ${ }^{9}$, Lars A. Buchhave ${ }^{3,10}$, Timothy R. Bedding ${ }^{6,5}$, Guy R. Davies ${ }^{4,5}$, SaSkia HeKkeR ${ }^{11,5}$, \\ Hans Kueldsen $^{5}$, James S. Kuszlewicz ${ }^{4,5}$, Mark E. EveretT ${ }^{12}$, Steve B. Howell ${ }^{13}$, Sarbani Basu ${ }^{14}$, Tiago L. Campante ${ }^{4,5}$, \\ Jørgen Christensen-DalsgaArd ${ }^{5}$, Yvonne P. Elsworth ${ }^{4,5}$, Christoffer KarofF ${ }^{5,15}$, Steven D. Kawaler ${ }^{16}$, \\ Mikkel N. Lund ${ }^{5,4}$, Mia Lundkvist ${ }^{5}$, Gilbert A. Esquerdo ${ }^{3}$, Michael L. Calkins ${ }^{3}$, and Perry Berlind ${ }^{3}$

\section{ABSTRACT}

We report the discovery of Kepler $-432 \mathrm{~b}$, a giant planet $\left(M_{\mathrm{b}}=5.41_{-0.18}^{+0.32} M_{\mathrm{Jup}}, R_{\mathrm{b}}=1.145_{-0.039}^{+0.036} R_{\mathrm{Jup}}\right)$ transiting an evolved star $\left(M_{\star}=1.32_{-0.07}^{+0.10} M_{\odot}, R_{\star}=4.06_{-0.08}^{+0.12} R_{\odot}\right)$ with an orbital period of $P_{\mathrm{b}}=52.501129_{-0.000053}^{+0.00067}$ days. Radial velocities (RVs) reveal that Kepler-432b orbits its parent star with an eccentricity of $e=0.5134_{-0.0089}^{+0.0098}$, which we also measure independently with asterodensity profiling (AP; $e=0.507_{-0.114}^{+0.039}$ ), thereby confirming the validity of AP on this particular evolved star. The well-determined planetary properties and unusually large mass also make this planet an important benchmark for theoretical models of super-Jupiter formation. Long-term RV monitoring detected the presence of a non-transiting outer planet (Kepler-432c; $M_{\mathrm{c}} \sin i_{\mathrm{c}}=2.43_{-0.24}^{+0.22} M_{\mathrm{Jup}}$, $P_{\mathrm{c}}=406.2_{-2.5}^{+3.9}$ days), and adaptive optics imaging revealed a nearby $(0.87)$, faint companion (Kepler $\left.-432 \mathrm{~B}\right)$ that is a physically bound $\mathrm{M}$ dwarf. The host star exhibits high signal-to-noise ratio asteroseismic oscillations, which enable precise measurements of the stellar mass, radius, and age. Analysis of the rotational splitting of the oscillation modes additionally reveals the stellar spin axis to be nearly edge-on, which suggests that the stellar spin is likely well aligned with the orbit of the transiting planet. Despite its long period, the obliquity of the 52.5 day orbit may have been shaped by star-planet interaction in a manner similar to hot Jupiter systems, and we present observational and theoretical evidence to support this scenario. Finally, as a short-period outlier among giant planets orbiting giant stars, study of Kepler-432b may help explain the distribution of massive planets orbiting giant stars interior to $1 \mathrm{AU}$.

Key words: asteroseismology - planets and satellites: dynamical evolution and stability - planets and satellites: formation - planets and satellites: gaseous planets - planet-star interactions - stars: individual (Kepler-432)

\section{INTRODUCTION}

The NASA Kepler mission (Borucki et al. 2010), at its heart a statistical endeavor, has provided a rich data set that enables ensemble studies of planetary populations, from gas giants to Earth-sized planets. Such investigations can yield valuable statistical constraints for theories of planetary formation and subsequent dynamical evolution (e.g., Buchhave et al. 2014; Steffen et al. 2012). Individual discoveries, however, provide important case studies to explore these processes in detail, especially in parameter space for which populations remain small. Because of its unprecedented photometric sensitivity,

\footnotetext{
${ }^{17}$ NASA Carl Sagan Fellow.

18 NSF Graduate Research Fellow.
}

duty cycle, and time coverage, companions that are intrinsically rare or otherwise difficult to detect are expected to be found by Kepler, and detailed study of such discoveries can lead to characterization of poorly understood classes of objects and physical processes.

Planets orbiting red giants are of interest because they trace the planetary population around their progenitors, many of which are massive and can be hard to survey while they reside on the main sequence. Stars more massive than about $1.3 M_{\odot}$ (the so-called Kraft break; Kraft 1967) have negligible convective envelopes, which prevents the generation of the magnetic winds that drive angular momentum loss in smaller stars. Their rapid rotation and high temperatures-resulting in broad spectral features that are sparse in the optical-make 
precise radial velocities (RVs) extremely difficult with current techniques. However, as they evolve to become giant stars, they cool and spin down, making them ideal targets for precise radial velocity work (see, e.g., Johnson et al. 2011, and references therein). There is already good evidence that planetary populations around intermediate-mass stars are substantially different from those around their low-mass counterparts. Higher mass stars seem to harbor more Jupiters than do Sun-like stars (Johnson et al. 2010), and the typical planetary mass correlates with the stellar mass (Lovis \& Mayor 2007; Döllinger et al. 2009; Bowler et al. 2010), but there are not many planets within 1 AU of more massive stars (Johnson et al. 2007; Sato et al. 2008), and their orbits tend to be less eccentric than Jupiters orbiting low-mass stars (Jones et al. 2014). Due to the observational difficulties associated with massive and intermediate-mass main-sequence stars, many of the more massive stars known to host planets have already reached an advanced evolutionary state, and it is not yet clear whether most of the orbital differences can be attributed to mass-dependent formation and migration, or if planetary engulfment and/or tidal evolution as the star swells on the giant branch plays a more important role.

While the number of planets known to orbit evolved stars has become substantial, because of their typically long periods, not many transit, and thus very few are amenable to detailed study. In fact, Kepler-91b (Lillo-Box et al. 2014b, 2014a; Barclay et al. 2014) and Kepler-56c (Huber et al. 2013b) are the only two massive planets $\left(M_{p}>0.5 M_{\text {Jup }}\right)$ orbiting giant stars $(\log g<3.9)$ that are known to transit. ${ }^{19}$ A transit leads to a radius measurement, enabling investigation of interior structure and composition via bulk density constraints and theoretical models (e.g., Fortney et al. 2007), and also opens up the possibility of atmospheric studies (e.g., Charbonneau et al. 2002; Knutson et al. 2008; Berta et al. 2012; Poppenhaeger et al. 2013), which can yield more specific details about planetary structure, weather, or atomic and molecular abundances within the atmosphere of the planet. Such information can provide additional clues about the process of planet formation around hot stars.

In studies of orbital migration of giant planets, the stellar obliquity - the angle between the stellar spin axis and the orbital angular momentum vector-has proven to be a valuable measurement, as it holds clues about the dynamical history of the planetary system (see, e.g., Albrecht et al. 2012). Assuming that the protoplanetary disk is coplanar with the stellar equator, and thus the rotational and orbital angular momenta start out well aligned, some migration mechanisms-e.g., Type II migration (Goldreich \& Tremaine 1980; Lin \& Papaloizou 1986)—are expected to preserve low obliquities, while others-for example, Kozai cycles (e.g., Wu \& Murray 2003; Fabrycky \& Tremaine 2007), secular chaos (Wu \& Lithwick 2011), or planet-planet scattering (e.g., Rasio \& Ford 1996; Juric \& Tremaine 2008) - may excite large orbital inclinations. Measurements of stellar obliquity can thus potentially distinguish between classes of planetary migration.

The Rossiter-McLaughlin effect (McLaughlin 1924; Rossiter 1924) has been the main source of (projected) obliquity measurements, although at various times starspot crossings (e.g., Désert et al. 2011; Nutzman et al. 2011; Sanchis-Ojeda et al. 2011), Doppler tomography of planetary

\footnotetext{
19 Among the other 95 such planets listed in The Exoplanet Orbit Database (exoplanets.org), none transit.
}

transits (e.g., Collier Cameron et al. 2010; Johnson et al. 2014), gravity-darkened models of the stellar disk during transit (e.g., Barnes et al. 2011), and the rotational splitting of asteroseismic oscillation modes (e.g., Huber et al. 2013b) have all been used to determine absolute or projected stellar obliquities. Most obliquity measurements to date have been for hot Jupiters orbiting Sun-like stars, but to get a full picture of planetary migration, we must study the dynamical histories of planets across a range of separations and in a variety of environments. Luckily, the diversity of techniques with which we can gather this information allows us to begin investigating spin-orbit misalignment for long-period planets and around stars of various masses and evolutionary states. For long-period planets orbiting slowly rotating giant stars, the Rossiter-McLaughlin amplitudes are small because they scale with the stellar rotational velocity and the planet-to-star area ratio, and few transits occur, which limits the opportunities to obtain follow-up measurements or to identify the transit geometry from spot crossings. Fortunately, the detection of asteroseismic modes does not require rapid rotation and is independent of the planetary properties, so it becomes a valuable tool for long-period planets orbiting evolved stars. The high-precision, high-duty-cycle, longtimespan, photometric observations of Kepler are ideal for both identifying long-period transiting planets and examining the asteroseismic properties of their host stars.

In this paper, we highlight the discovery of Kepler- $432 \mathrm{~b}$ and $\mathrm{c}$, a pair of giant planets in long-period orbits ( $>50$ days) around an oscillating, intermediate-mass red giant. We present the photometric observations and transit light-curve analysis of Kepler-432 in Section 2 and the follow-up imaging and spectroscopy in Sections 3 and 4, followed by the asteroseismic and radial velocity analyses in Sections 5 and 6 . False positive scenarios and orbital stability are investigated in Sections 7 and 8 , and we discuss the system in the context of planet formation and migration in Section 9, paying particular attention to starplanet interactions (SPIs) and orbital evolution during the red giant phase. We provide a summary in Section 10.

\section{PHOTOMETRY}

\subsection{Kepler Observations}

The Kepler mission and its photometric performance are described in Borucki et al. (2010), and the characteristics of the detector on board the spacecraft are described in Koch et al. (2010) and van Cleve (2008). The photometric observations of Kepler-432 span Kepler observation Quarters 0 through 17 (JD 2454953.5 to 2456424.0 ), a total of 1470.5 days. Kepler432 b was published by the Kepler team as a Kepler Object of Interest (KOI) and planetary candidate (designated KOI-1299; see Batalha et al. 2013), and after also being identified as a promising asteroseismic target, it was observed in shortcadence (SC) mode for 8 quarters. We note that a pair of recent papers have now confirmed the planetary nature of this transiting companion via radial velocity measurements (Ciceri et al. 2015; Ortiz et al. 2015).

The full photometric time series, normalized in each quarter, is shown in Figure 1. A transit signature with a period of $\sim 52.5$ days is apparent in the data, and our investigation of the transits is described in the following section. 


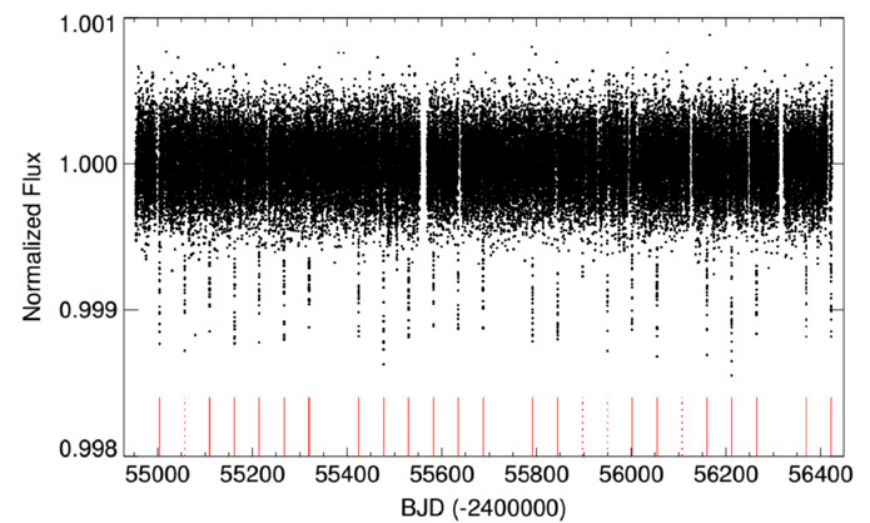

Figure 1. Detrended and normalized long-cadence Kepler time series for Kepler-432, spanning 1470.5 days. The transits, which are clearly visible even in the unfolded data, are indicated by red lines. Solid lines denote full transits, while dotted lines denote that only a partial transit was observed. Three of the 28 expected transits occurred entirely during data gaps.

\subsection{Light-curve Analysis}

A transit light-curve analysis of Kepler-432b was performed previously by Sliski \& Kipping (2014). In that work, the authors first detrended the Simple Aperture Photometry (SAP) Kepler data $^{20}$ for quarters 1-17 using the CoFiAM (Cosine Filtering with Autocorrelation Minimization) algorithm and then regressed the cleaned data with the multimodal nested sampling algorithm MultiNest (Feroz et al. 2009) coupled to a Mandel \& Agol (2002) planetary transit model. Details on the priors employed and treatment of limb darkening are described in Sliski \& Kipping (2014). The authors compared the light-curve derived stellar density, $\rho_{\star \text { obs }}$, to that from asteroseismology, $\rho_{\star \text {,astero }}$, in a procedure dubbed "Asterodensity Profiling" (AP; Kipping et al. 2012; Kipping 2014a) to constrain the planet's minimum orbital eccentricity as being $e_{\min }=0.488_{-0.051}^{+0.025}$. The minimum eccentricity is most easily retrieved with AP, but the proper eccentricity (and argument of periastron, $\omega$ ) can be estimated by including $e$ and $\omega$ as free parameters in the lightcurve fit and marginalizing over $\omega$. In order to estimate the proper eccentricity, we were motivated to re-visit the Kepler data, as described below.

We first detrended the Kepler SAP data as was done in Sliski \& Kipping (2014), by using the CoFiAM algorithm, which is described in detail in Kipping et al. (2013). CoFiAM acts like a harmonic filter, removing any long-term periodicities in the data but protecting those variations occurring on the timescale of the transit or shorter, so as to retain the true light-curve shape. The algorithm requires an estimate of the times of transit minimum, orbital period, and full transit duration. Since Sliski \& Kipping (2014) provided refined values for these quantities, we used these updated values to conduct a revised CoFiAM detrending of the Kepler data. As with the previous analysis, the final light-curves are optimized for a window within three transit durations of the transit minima.

Due to the effects of stellar granulation on the photometry, we find that the light-curve scatter clearly exceeds the typical photometric uncertainties. In order to obtain more realistic parameter uncertainties, we added a "jitter" term in quadrature to the photometric uncertainties to yield a reduced chi-squared

\footnotetext{
${ }^{20}$ Observations labeled as SAP FLUX in FITS files retrieved from the Barbara A. Mikulski Archive for $\bar{S}$ pace Telescopes (MAST).
}

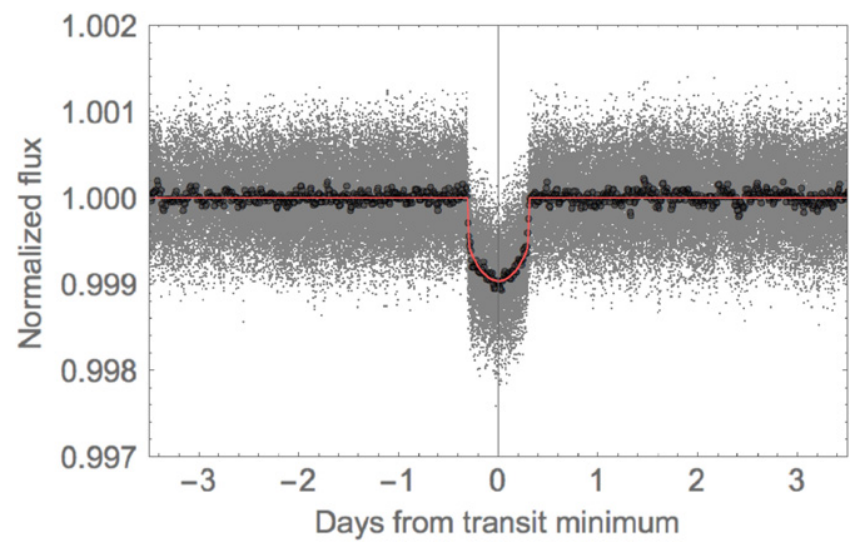

Figure 2. Folded short-cadence Kepler light-curve, shown as gray points. For clarity, the binned data (every 100 points) are overplotted as large dark circles, and the best fit, with parameters reported in Table 1 , is indicated by the solid red line.

of unity for the out-of-transit data. This was done independently for the long- and short-cadence data, although the photometric jitter terms were (as expected) nearly identical at 177.4 and $175.9 \mathrm{ppm}$ for the short- and long-cadence data, respectively.

The 13 long-cadence transits for which no SC data were available and the $11 \mathrm{SC}$ transits were stitched together and regressed to a transit model using MultiNest. Our light-curve model employs the quadratic limb darkening Mandel \& Agol (2002) routine with the Kipping (2010) "resampling" prescription for accounting for the smearing of the long-cadence data. The seven basic parameters in our light-curve fit were ratio-of-radii, $R_{\mathrm{b}} / R_{\star}$, stellar density, $\rho_{\star}$, impact parameter, $b$, time of transit minimum, $T_{0}$, orbital period, $P$, and the limbdarkening coefficients $q_{1}$ and $q_{2}$ described in Kipping (2013a). In addition, we included an eighth parameter for the log of the contaminated light fraction from a blend source, $\log _{10} \beta=\log _{10}\left(F_{\text {blend }} / F_{\star}\right)$. This was constrained from adaptive optics imaging (AO; see Section 3.2) to be $\log \beta=-2.647 \pm 0.042$ with a Gaussian prior, assuming Gaussian uncertainties on the magnitudes measured from AO.

Ordinarily, a transit light-curve contains very little information on the orbital eccentricity and thus it is not possible to reach a converged eccentricity solution with photometry alone (Kipping 2008). However, in cases where the parent star's mean density is independently constrained, a transit light-curve can be used to constrain the orbital eccentricity and argument of periastron (Dawson \& Johnson 2012; Kipping 2014a). This technique, an example of AP, enables us to include $e$ and $\omega$ as our ninth and 10th transit model parameters.

To enable the use of AP, we impose an informative Gaussian prior on the mean stellar density given by the asteroseismology constraint $\left(\rho_{\star \text {,astero }}=27.94_{-0.58}^{+0.55} \mathrm{~kg} \mathrm{~m}^{-3}\right.$; Section 5$)$. We use the ECCSAMPLES code (Kipping 2014b) to draw samples from an appropriate joint $e-\omega$ prior. This code describes the eccentricity distribution as following a Beta distribution (a weakly informative prior) and then accounts for the bias in both $e$ and $\omega$ caused by the fact that the planet is known to transit. For the Beta distribution shape parameters, we use the "short"-period calibration $(P<380$ days) of Kipping $(2013 b)$ : $a_{\beta}=0.697$ and $b_{\beta}=3.27$.

The maximum a posteriori folded transit light-curve is presented in Figure 2. The transit parameters and associated 
Table 1

Kepler-432 Transit Parameters

\begin{tabular}{lr}
\hline \hline Parameter & Value \\
\hline$R_{\mathrm{b}} / R_{\star}$ & $0.02914_{-0.00093}^{+0.00038}$ \\
$\rho_{\star}\left(\mathrm{kg} \mathrm{m}^{-3}\right)$ & $27.94_{-0.58}^{+0.54}$ \\
$b$ & $0.503_{-0.168}^{+0.090}$ \\
$T_{0, \mathrm{~b}}(\mathrm{BJD})$ & $2455949.5374_{-0.0016}^{+0.0018}$ \\
$P_{\mathrm{b}}($ days $)$ & $52.501134_{-0.000107}^{+0.000070}$ \\
$q_{1}$ & $0.309_{-0.042}^{+0.047}$ \\
$q_{2}$ & $0.674_{-0.098}^{+0.151}$ \\
$\log \beta$ & $-2.647 \pm 0.042$ \\
$e_{\mathrm{b}}$ & $0.507_{-0.114}^{+0.039}$ \\
$\omega_{\mathrm{b}}($ degrees $)$ & $76_{-24}^{+59}$ \\
\hline
\end{tabular}

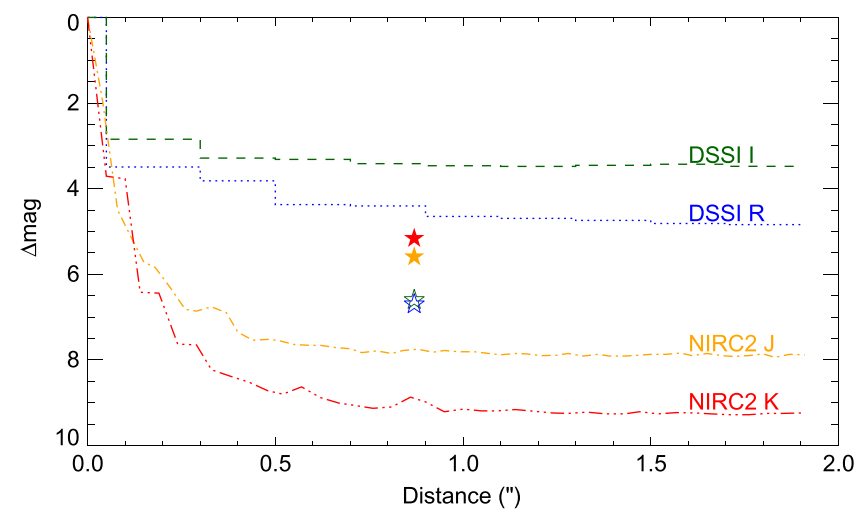

Figure 3. Contrasts as a function of distance for all high-resolution follow-up, and the magnitude difference of the visual companion in each filter. Based on its infrared magnitudes from the NIRC2 $J$ - and $K$-band detections (filled stars), we estimate the companion contrast in the $R$ - and $I$-bands (open stars) to be 6.7 and $6.6 \mathrm{mag}$, respectively. These magnitudes are consistent with our nondetections in the DSSI images.

68.3\% uncertainties, derived solely from this photometric fit, are reported in Table 1.

\section{HIGH SPATIAL RESOLUTION IMAGING}

\subsection{Speckle Imaging}

Speckle imaging observations of Kepler-432 were performed on UT 2011 June 16 at the $3.5 \mathrm{~m}$ WIYN telescope on Kitt Peak, AZ, using the Differential Speckle Survey Instrument (DSSI; Horch et al. 2010). DSSI provides simultaneous images in two filters using a dichroic beam splitter and two identical EMCCDs. These images were obtained in the $R(6920 \AA)$ and $I(8800 \AA)$ bands. Data reduction and analysis of these images are described in Torres et al. (2011), Horch et al. (2010), and Howell et al. (2011). The reconstructed $R$ - and $I$ band images reveal no stellar companions brighter than $\Delta R \sim 4.5 \mathrm{mag}$ and $\Delta I \sim 3.5 \mathrm{mag}$, within the annulus from 0 . 05 to $2^{\prime \prime}$. The contrasts achieved as a function of distance are plotted in Figure 3 and represent $5 \sigma$ detection thresholds.

\subsection{Adaptive Optics Imaging}

Adaptive optics imaging was obtained using the Near InfraRed Camera 2 (NIRC2) mounted on the Keck II $10 \mathrm{~m}$ telescope on Mauna Kea, HI, on UT 2014 September 4. Images were obtained in both $J(1.260 \mu \mathrm{m})$ and $\operatorname{Br} \gamma(2.165 \mu \mathrm{m}$; a good proxy for both $K$ and $K_{s}$ ). NIRC2 has a field of view of $10^{\prime \prime} \times 10^{\prime \prime}$, a pixel scale of about $0 . \prime 01$ pixel $^{-1}$, and a rotator accuracy of 0.02 . The overlap region of the dither pattern of the observations (i.e., the size of the final combined images) is $\sim 4^{\prime \prime} \times 4^{\prime \prime}$. In both filters, the FWHM of the stellar point-spread function was better than $0 . \prime 05$, and the achieved contrasts were $\Delta K \sim 9(\Delta J \sim 8)$ beyond $0 . " 5$ (see Figure 3).

A visual companion was detected in both images (Figure 4) with separation $0 .{ }^{\prime \prime} 8730 \pm 0 . \prime 0014$ and PA 20:86 \pm 0.07 (east of north). Relative to Kepler-432, we calculate the companion to have magnitudes $\Delta J=5.59 \pm 0.06$ and $\Delta K=5.16 \pm 0.02$, implying $J-K=0.99 \pm 0.07$. Using the $J-K$ colors, we estimate the magnitude in the Kepler bandpass to be $K_{\mathrm{p}} \sim 18.8$. The object was not detected in the speckle images because they were taken with less aperture and the expected contrast ratios are larger in $R$ and $I$-using the properties of the companion as derived in the following section, we estimate $\Delta R \sim 6.7$ and $\Delta I \sim 6.6$. These magnitudes are consistent with non-detections in the speckle images, as plotted in Figure 3.

\subsection{Properties of the Visual Companion}

The faint visual companion to Kepler-432 could be a background star or a physically bound main-sequence companion. We argue that it is unlikely to be a background star and present two pieces of evidence to support this conclusion. We first estimate the background stellar density in the direction of Kepler-432 using the TRILEGAL stellar population synthesis tool (Girardi et al. 2005): we expect 49,000 sources per $\operatorname{deg}^{2}$ that are brighter than $K_{s} \sim 19$ (the detection limit of our observation). This translates to 0.06 sources (of any brightness and color) expected in our $16 \operatorname{arcsec}^{2}$ image, and thus the a priori probability of a chance alignment is low.

Furthermore, since we know the properties of the primary star, we can determine whether there exists a coeval mainsequence star that could adequately produce the observed colors and magnitude differences. Using the asteroseismically derived mass, radius, and age of the primary (see Section 5), we place the primary star on an appropriate Padova PARSEC isochrone (Bressan et al. 2012; Chen et al. 2014). We then use the observed magnitude differences between the stars to search for an appropriate match to the companion in the isochrone. If the visual companion is actually a background giant, it is unlikely that it would happen to be at the right distance to match both the colors and brightness of a physically bound companion. Therefore, we do not expect a background giant star to lie on the isochrone. However, we do find a close match to the observed colors and magnitudes of the companion (see Figure 5), further suggesting that it is not a background object, but truly a physical companion, and this allows us to estimate its properties from the isochrone.

We conclude that the companion is most likely a physically bound, coeval $\mathrm{M}$ dwarf with a mass of $\sim 0.52 M_{\odot}$ and an effective temperature of $\sim 3660 \mathrm{~K}$. The distance to the system $(\sim 870 \mathrm{pc}$; Section 5.5$)$ implies that the projected separation of the companion is $\sim 750 \mathrm{AU}$. Using this as an estimate of the semi-major axis, the binary orbital period is on the order of $15,000 \mathrm{yr}$. In reality, the semi-major axis may be smaller (if we observed it near apastron of an eccentric orbit with the major axis in the plane of the sky), or significantly larger (due to 

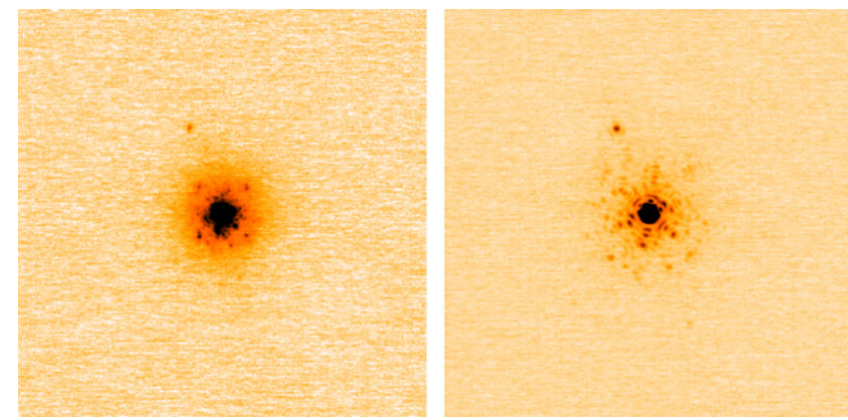

Figure 4. NIRC2 AO images in $J$-band $(1.260 \mu \mathrm{m})$ and $\operatorname{Br} \gamma(2.165 \mu \mathrm{m})$, $4^{\prime \prime} \times 4^{\prime \prime}$ in size, with a logarithmic flux scale. A faint companion to the northeast is clearly detected in both images, with separation 0 " $8730 \pm 0$ ".0014 and PA $20: 86 \pm 0: 07$. North is up and east is left.

projections into the plane of the sky and the unknown orbital phase).

We discuss the possibility of false positives due to this previously undetected companion in Section 7.

\section{SPECTROSCOPIC FOLLOW-UP}

\subsection{Spectroscopic Observations}

We used the Tillinghast Reflector Echelle Spectrograph (TRES; Fûrész 2008) mounted on the $1.5 \mathrm{~m}$ Tillinghast Reflector at the Fred L. Whipple Observatory (FLWO) on Mt. Hopkins, AZ, to obtain 84 high-resolution spectra of Kepler-432 between UT 2011 March 23 and 2014 June 18. TRES is a temperature-controlled, fiber-fed instrument with a resolving power of $R \sim 44,000$ and a wavelength coverage of 3850-9100 A, spanning 51 echelle orders. Typical exposure times were 15-30 minutes and resulted in extracted signal-tonoise ratios (S/Ns) between about 20 and 45 per resolution element. The goal of the intial observations was to rule out false positives involving stellar binaries as part of the Kepler Follow-up Observing Program (KFOP, which has evolved into $\mathrm{CFOP}^{21}$ ), but upon analysis of the first few spectra, it became clear that the planet was massive enough to confirm with an instrument like TRES that has a modest aperture and $\sim 10 \mathrm{~m} \mathrm{~s}^{-1}$ precision (see, e.g., Quinn et al. 2014). By the second observing season, an additional velocity trend was observed, which led to an extended campaign of observations.

Precise wavelength calibration of the spectra was established by obtaining ThAr emission-line spectra before and after each spectrum, through the same fiber as the science exposures. Nightly observations of the IAU RV standard star HD 182488 helped us track the achieved instrumental precision and correct for any RV zero-point drift. We also shift the absolute velocities from each run so that the median $\mathrm{RV}$ of HD 182488 is $-21.508 \mathrm{~km} \mathrm{~s}^{-1}$ (Nidever et al. 2002). This allows us to report the absolute systemic velocity, $\gamma_{\mathrm{abs}}$. We are aware of specific TRES hardware malfunctions (and upgrades) that occurred during the time span of our data that, in addition to small zero-point shifts (typically $<10 \mathrm{~m} \mathrm{~s}^{-1}$ ), caused degradation (or improvement) of RV precision for particular observing runs. For example, the installation of a new dewar

\footnotetext{
21 The Kepler Community Follow-up Observing Program, CFOP, http://cfop. ipac.caltech.edu, publicly hosts spectra, images, data analysis products, and observing notes for Kepler-432 and many other Kepler Objects of Interest (KOIs).
}

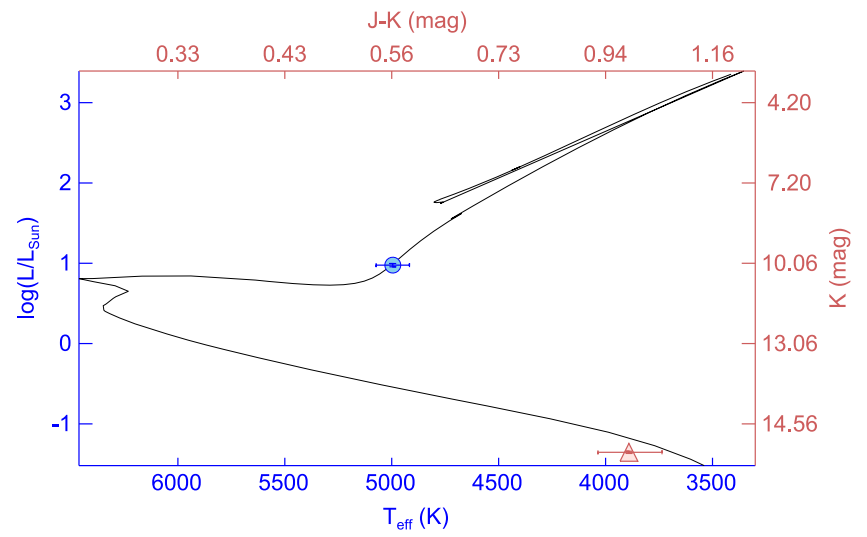

Figure 5. Kepler-432 (blue circle) placed on a $3.5 \mathrm{Gyr},[\mathrm{m} / \mathrm{H}]=-0.07$ PARSEC isochrone (Bressan et al. 2012), plotted as a solid black line. The upper right (red) axes represent the $J-K$ and apparent $K$ magnitude corresponding to the $T_{\text {eff }}$ and $\log L / L \odot$ on the lower left (blue) axes. The visual companion (red triangle) is placed on the plot according to its measured $J-K$ and $K$ magnitude. It lies very near the isochrone for the system, suggesting that it is indeed coeval with and physically bound to Kepler-432, rather than a background star.

lens caused a zero-point shift after BJD 2,455,750, and a second shift (accompanied by significant improvement in precision) occurred when the fiber positioner was fixed in place on BJD 2,456,013. It will be important to treat these with care so that the radial velocities are accurate and each receives its appropriate weight in our analysis.

We also obtained five spectra with the FIber-fed Echelle Spectrograph (FIES; Frandsen et al. 1999) on the $2.5 \mathrm{~m}$ Nordic Optical Telescope (NOT; Djupvik et al. 2010, p. 211) at La Palma, Spain, during the first observing season (UT 2011 August 4 through 2011 October 7) to confirm the initial RV detection before continuing to monitor the star with TRES. Like TRES, FIES is a temperature-controlled, fiber-fed instrument and has a resolving power through the medium fiber of $R \sim 46,000$, a wavelength coverage of $\sim 3600-7400 \AA$, and wavelength calibration determined from ThAr emissionline spectra.

\subsection{Spectroscopic Reduction and Radial Velocity Determination}

We will discuss the reduction of spectra from both instruments collectively but only briefly (more details can be found in Buchhave et al. 2010) while detailing the challenges presented by our particular data set. Spectra were optimally extracted, rectified to intensity versus wavelength, and crosscorrelated, order by order, using the strongest exposure as a template. We used 21 orders (spanning 4290-6280 A), rejecting those plagued by telluric lines, fringing in the red, and low $\mathrm{S} / \mathrm{N}$ in the blue. For each epoch, the cross-correlation functions (CCFs) from all orders were added and fit with a Gaussian to determine the relative RV for that epoch. Using the summed $\mathrm{CCF}$ rather than the mean of RVs from each order naturally weights the orders with high correlation coefficients more strongly. Internal error estimates for each observation were calculated as $\sigma_{\text {int }}=\operatorname{rms}(v) / \sqrt{N}$, where $v$ is the RV of each order, $N$ is the number of orders, and rms denotes the root mean squared velocity difference from the mean. These internal errors account for photon noise and the precision with which we can measure the line centers, which in turn depends on the characteristics of the Kepler-432 spectrum (line shapes, 
number of lines, etc.), but do not account for errors introduced by the instrument itself.

The nightly observations of RV standards were used to correct for systematic velocity shifts between runs and to estimate the instrumental precision. The median RV of HD 182488 was calculated for each run, which we applied as shifts to the Kepler432 velocities, keeping in mind that each shift introduces additional uncertainty. By also applying the run-to-run offsets to the standard star RVs themselves, we were able to evaluate the residual RV noise introduced by the limited instrumental precision (separate from systematic zero-point shifts). After correction, the rms of the standard star RVs in each run was consistent with the internal errors. This indicates that the additional uncertainty introduced by run-to-run correction already adequately accounts for the instrumental uncertainty, and we do not need to explicitly include an additional error term to account for it. The final error budget of Kepler-432 RVs was assumed to be the sum by quadrature of all RV error sourcesinternal errors, run-to-run offset uncertainties, and TRES instrumental precision: $\sigma_{\mathrm{RV}}^{2}=\sigma_{\text {int }}^{2}+\sigma_{\text {run }}^{2}+\sigma_{\text {TRES }}^{2}$, where the final term $\sigma_{\mathrm{TRES}}=0$ because it is implicitly incorporated into $\sigma_{\text {run }}$. The final radial velocities are listed in Table 2. We recognize that stellar jitter or additional undetected planets may also act as noise sources, and we address this during the orbital fitting analysis in Section 6.

\subsection{Spectroscopic Classification}

We initially determined the spectroscopic stellar properties (effective temperature, $T_{\text {eff }}$; surface gravity, $\log g$; projected rotational velocity, $v \sin i$; and metallicity, $[\mathrm{m} / \mathrm{H}])$ using Stellar Parameter Classification (SPC; Buchhave et al. 2012), with the goal of providing an accurate temperature for the asteroseismic modeling (see Section 5). SPC cross-correlates an observed spectrum against a grid of synthetic spectra and uses the correlation peak heights to fit a three-dimensional surface in order to find the best combination of atmospheric parameters ( $v \sin i$ is fit iteratively since it only weakly correlates with the other parameters). We used the CfA library of synthetic spectra, which are based on Kurucz model atmospheres (Kurucz 1992). SPC, like other spectroscopic classifications, can be limited by degeneracy between $T_{\text {eff }}, \log g$, and $[\mathrm{m} / \mathrm{H}]$ (see a discussion in Torres et al. 2012), but asteroseismology provides a nearly independent measure of the surface gravity (depending only weakly on the effective temperature and metallicity). This allows one to iterate the two analyses until agreement is reached, generally requiring only 1 iteration (see, e.g., Huber et al. 2013a). In our initial analysis, we found $T_{\text {eff }}=5072 \pm 55 \mathrm{~K}$, $\log g=3.49 \pm 0.11,[\mathrm{~m} / \mathrm{H}]=-0.02 \pm 0.08$, and $v \sin i=$ $2.5 \pm 0.5 \mathrm{~km} \mathrm{~s}^{-1}$. After iterating with the asteroseismic analysis and fixing the final asteroseismic gravity, we find similar values: $\mathrm{T}_{\mathrm{eff}}=4995 \pm 78 \mathrm{~K}, \log g=3.345 \pm 0.006,[\mathrm{~m} / \mathrm{H}]=-0.07$ \pm 0.10 , and $v \sin i=2.7 \pm 0.5 \mathrm{~km} \mathrm{~s}^{-1}$. We adopt the values from the combined analysis, and these final spectroscopic parameters are listed in Table 3.

\section{ASTEROSEISMOLOGY OF KEPLER-432}

\subsection{Background}

Cool stars exhibit brightness variations due to oscillations driven by near-surface convection (Houdek et al. 1999; Aerts et al. 2010), which are a powerful tool to study their density profiles and evolutionary states. A simple asteroseismic
Table 2

Kepler-432 Relative Radial Velocities

\begin{tabular}{|c|c|c|c|c|c|}
\hline $\begin{array}{l}\text { BJD } \\
(-2,455,000)\end{array}$ & $\begin{array}{c}v \\
\left(\mathrm{~ms}^{-1}\right)\end{array}$ & $\begin{array}{c}\sigma_{v}{ }^{\mathrm{a}} \\
\left(\mathrm{ms}^{-1}\right)\end{array}$ & $\begin{array}{c}\text { BJD } \\
(-2,455,000)\end{array}$ & $\begin{array}{c}v \\
\left(\mathrm{~ms}^{-1}\right)\end{array}$ & $\begin{array}{c}\sigma_{v}^{\mathrm{a}} \\
\left(\mathrm{ms}^{-1}\right)\end{array}$ \\
\hline 644.00217 & 235.4 & 65.1 & 1083.87676 & 338.7 & 36.7 \\
\hline 722.95848 & 453.1 & 23.0 & 1091.93504 & 489.2 & 44.3 \\
\hline 727.87487 & 452.2 & 72.1 & 1117.90004 & 211.7 & 41.6 \\
\hline 734.86675 & 544.4 & 68.2 & 1132.79275 & 410.9 & 42.4 \\
\hline 755.93317 & 47.2 & 18.9 & 1137.83647 & 333.7 & 21.8 \\
\hline 757.82852 & 197.1 & 23.7 & 1175.82536 & 210.1 & 62.5 \\
\hline 758.88922 & 121.7 & 18.9 & 1197.74504 & 394.7 & 22.3 \\
\hline 760.95496 & 193.8 & 25.9 & 1202.70980 & 495.3 & 20.5 \\
\hline 764.92787 & 270.3 & 36.7 & 1227.64276 & 75.4 & 23.1 \\
\hline 768.78698 & 262.1 & 33.9 & 1233.59012 & 213.1 & 32.0 \\
\hline 768.80782 & 277.4 & 30.8 & 1258.59157 & 564.4 & 24.3 \\
\hline 822.70873 & 197.0 & 54.9 & 1279.58992 & 96.6 & 28.9 \\
\hline 825.63058 & 265.0 & 53.1 & 1376.99835 & 29.1 & 25.1 \\
\hline 826.64436 & 310.1 & 53.0 & 1382.93180 & 148.7 & 23.9 \\
\hline 827.71851 & 362.0 & 55.3 & 1389.91148 & 179.3 & 26.7 \\
\hline 828.61567 & 358.8 & 60.8 & 1400.93674 & 384.5 & 31.0 \\
\hline 829.72558 & 358.7 & 53.9 & 1405.90249 & 465.6 & 30.5 \\
\hline 830.69401 & 446.6 & 56.2 & 1409.87680 & 507.5 & 28.4 \\
\hline 837.59392 & 588.0 & 51.1 & 1413.96428 & 646.1 & 37.2 \\
\hline 840.65649 & 603.7 & 51.0 & 1429.95861 & 111.4 & 23.7 \\
\hline 841.70196 & 706.7 & 71.9 & 1436.83822 & 153.9 & 22.7 \\
\hline 842.57863 & 574.4 & 57.1 & 1442.84894 & 191.6 & 26.9 \\
\hline 843.59367 & 437.4 & 43.8 & 1446.78723 & 285.9 & 21.2 \\
\hline 844.64075 & 346.9 & 46.7 & 1503.72052 & 351.9 & 35.0 \\
\hline 845.68516 & 157.7 & 45.8 & 1547.69956 & 189.1 & 19.5 \\
\hline 846.62110 & 91.9 & 45.3 & 1551.63468 & 217.2 & 20.0 \\
\hline 852.61538 & 64.2 & 47.3 & 1556.67843 & 292.6 & 20.9 \\
\hline 854.62438 & 57.6 & 48.9 & 1561.63231 & 370.3 & 18.2 \\
\hline 856.71790 & 132.6 & 56.1 & 1575.69266 & 593.5 & 20.0 \\
\hline 858.64825 & 105.2 & 48.9 & 1581.66560 & 141.4 & 18.9 \\
\hline 1027.94566 & 284.4 & 26.4 & 1586.63377 & 40.5 & 18.4 \\
\hline 1046.89934 & 594.1 & 20.6 & 1591.70580 & 100.0 & 21.4 \\
\hline 1047.87727 & 702.8 & 38.6 & 1729.01428 & 553.2 & 37.9 \\
\hline 1048.86080 & 693.7 & 18.9 & 1740.94781 & 98.6 & 33.0 \\
\hline 1049.97884 & 661.7 & 28.7 & 1743.99496 & 34.8 & 29.3 \\
\hline 1051.99004 & 631.1 & 24.6 & 1799.87657 & 37.0 & 26.3 \\
\hline 1052.93268 & 583.1 & 30.0 & 1816.87894 & 248.9 & 32.0 \\
\hline 1053.90973 & 461.0 & 23.4 & 1822.88725 & 364.1 & 24.3 \\
\hline 1054.88376 & 383.9 & 29.3 & 1826.79500 & 419.8 & 20.0 \\
\hline 1055.88175 & 282.3 & 37.3 & ${ }^{\mathrm{b}} 777.59554$ & -100.9 & 10.7 \\
\hline 1056.88078 & 239.3 & 26.4 & b778.59121 & -81.1 & 10.5 \\
\hline 1057.95303 & 158.7 & 24.6 & b779.59677 & -51.2 & 14.1 \\
\hline 1058.84781 & 160.9 & 33.7 & b782.57231 & 0.0 & 10.2 \\
\hline 1074.90585 & 267.8 & 31.1 & b842.41733 & 47.7 & 10.2 \\
\hline 1080.95245 & 354.5 & 30.6 & & & \\
\hline
\end{tabular}

${ }^{\mathrm{a}}$ Values reported here account for internal and instrumental error, as well as uncertainties in systematic zero-point shifts applied to the velocities, but do not include the $20 \mathrm{~m} \mathrm{~s}^{-1}$ added during the orbital fit that is meant to encompass additional astrophysical noise sources (e.g., stellar activity or additional planets).

${ }^{b}$ The FIES observations have a different zero point, which is included as a free parameter in the orbital fit.

analysis is based on the average separation of modes of equal spherical degree $(\Delta \nu)$ and the frequency of maximum oscillation power $\left(\nu_{\max }\right)$, using scaling relations to estimate the mean stellar density, surface gravity, radius, and mass (Kjeldsen \& Bedding 1995; Stello et al. 2008; Kallinger et al. 2010; Belkacem et al. 2011). Huber et al. (2013a) presented an asteroseismic analysis of Kepler- 432 by measuring $\Delta \nu$ and $\nu_{\max }$ using three quarters of SC data, combined with an SPC analysis (Buchhave et al. 2012) of high-resolution 
Table 3

Stellar Properties of Kepler-432

\begin{tabular}{lcc}
\hline \hline Parameter & \multicolumn{2}{c}{ Value } \\
\hline Asteroseismic & Grid-based Modeling & Frequency Modeling \\
\hline$\Delta \nu(\mu \mathrm{Hz})$ & $18.59 \pm 0.04$ & $\ldots$ \\
$\nu_{\max }(\mu \mathrm{Hz})$ & $266 \pm 3$ & $\ldots$ \\
$\log (g)(\mathrm{dex})$ & $3.340 \pm 0.006$ & $3.345 \pm 0.006$ \\
$\rho_{\star}\left(\mathrm{g} \mathrm{cm}^{-3}\right)$ & $0.02723_{-0.00057}^{+0.00054}$ & $0.02794_{-0.00058}^{+0.00055}$ \\
$R\left(R_{\odot}\right)$ & $4.12_{-0.08}^{+0.12}$ & $4.06_{-0.08}^{+0.12}$ \\
$M\left(M_{\odot}\right)$ & $1.35_{-0.07}^{+0.10}$ & $1.32_{-0.07}^{+0.10}$ \\
Age $(\mathrm{Gyr})$ & $3.5_{-0.8}^{+0.7}$ & $4.2_{-1.0}^{+0.8}$ \\
\hline
\end{tabular}

Spectroscopic

\begin{tabular}{lc}
\hline$T_{\text {eff }}(\mathrm{K})$ & $4995 \pm 78$ \\
$\log g(\mathrm{cgs})$ & $3.345 \pm 0.006$ \\
{$[m / H]$} & $-0.07 \pm 0.10$ \\
$v \sin i_{\star}\left(\mathrm{kms}^{-1}\right)$ & $2.7 \pm 0.5$ \\
\hline
\end{tabular}

Photometric

\begin{tabular}{ll}
\hline$V(\mathrm{mag})^{\mathrm{a}}$ & $12.465 \pm 0.060$ \\
$K_{\mathrm{p}}(\mathrm{mag})^{\mathrm{b}}$ & $12.183 \pm 0.020$ \\
$J(\mathrm{mag})^{\mathrm{c}}$ & $10.684 \pm 0.021$ \\
$H(\mathrm{mag})^{\mathrm{c}}$ & $10.221 \pm 0.019$ \\
$K_{\mathrm{s}}(\mathrm{mag})^{\mathrm{c}}$ & $10.121 \pm 0.017$
\end{tabular}

Derived

\begin{tabular}{lc}
$L_{\star}\left(L_{\odot}\right)^{\mathrm{d}}$ & $9.206 \pm 0.010$ \\
$d(\mathrm{pc})$ & $870 \pm 20$ \\
$i_{\star}\left({ }^{\circ}\right)$ & $90_{-8}^{+0}$ \\
$P_{\text {rot }}$ (days) & $77 \pm 14$ \\
\hline
\end{tabular}

Note. While we have separated the stellar properties by observational technique, many of them are interdependent, for example, as described in Sections 4 and 5. We adopt the asteroseismic results from detailed frequency modeling (rather than the grid-based approach) in subsequent analyses.

${ }^{a}$ From APASS, via UCAC4 (Zacharias et al. 2013).

${ }^{\mathrm{b}}$ From the Kepler Input Catalog (Brown et al. 2011).

${ }^{\mathrm{c}}$ From 2MASS (Skrutskie et al. 2006).

${ }^{\mathrm{d}}$ Calculated using the relation $\left(\frac{L_{\star}}{L_{\odot}}\right)=\left(\frac{R_{\star}}{R_{\odot}}\right)^{2}\left(\frac{T_{\mathrm{eff}, \star}}{T_{\mathrm{eff}, \odot}}\right)^{4}$.

spectra obtained with the FIES and TRES spectrographs. The results showed that Kepler-432 is an evolved star just beginning to ascend the red giant branch (RGB), with a radius of $R=4.16 \pm 0.12 R_{\odot}$ and a mass of $1.35 \pm 0.10 M_{\odot}$ (Table 3 , Figure 5).

Compared to average oscillation properties, individual frequencies offer a greatly increased amount of information by probing the interior sound speed profile. In particular, evolved stars oscillate in mixed modes, which occur when pressure modes excited on the surface couple with gravity modes confined to the core (Aizenman et al. 1977). Mixed modes place tight constraints on fundamental properties such as stellar age and provide the possibility to probe the core structure and rotation (Bedding et al. 2011; Beck et al. 2012; Mosser et al. 2012b). Importantly, relative amplitudes of individual oscillation modes that are split by rotation can be used to infer the stellar line-of-sight inclination (Gizon \& Solanki 2003), providing valuable information on the orbital architectures of transiting exoplanet systems (e.g., Chaplin et al. 2013; Huber et al. 2013b; Benomar et al. 2014; Lund et al. 2014; Van Eylen et al. 2014). In the following section we expand on the initial asteroseismic analysis by Huber et al. (2013a) by performing a detailed individual frequency analysis based on all eight quarters (Q9-17) of Kepler shortcadence data.

\subsection{Frequency Analysis}

The time series was prepared for asteroseismic analysis from the raw Kepler target pixel data using the Kepler, Asteroseismic Science Operations, Center (KASOC) filter (Handberg \& Lund 2014). The KASOC filter removes instrumental and transit signals from the light-curve, which may produce spurious peaks in the frequency domain. The power spectrum, shown in Figure 6, was computed using a Lomb-Scargle periodogram (Lomb 1976; Scargle 1982) calibrated to satisfy Parseval's theorem.

The pattern of oscillation modes in the power spectrum is typical of red giants, with $\ell=0$ modes of consecutive order being approximately equally spaced by $\Delta \nu$, adjacent to $\ell=2$ modes. In addition to the $\ell=0,2$ pairs, several $\ell=1$ mixed modes are observed in each radial order that, on inspection, are the outer components of rotationally split triplets corresponding to the $m= \pm 1$ modes. This indicates that the star is seen equator-on (see Section 5.3).

The relative $p$ - and $g$-mode behavior of each mixed mode depends on the strength of the coupling between the oscillation cavities in the stellar core and envelope. Detecting the $\ell=1$ modes with the greatest g-mode character may be challenging because they have low amplitudes and overlap in frequency with the $\ell=0$ and $\ell=2$ modes. Adding to the possible confusion, mixed $\ell=2$ and $\ell=3$ modes may also be present, although the weaker coupling between the $p$ - and $g$-modes results in only the most p-like modes having an observable amplitude.

The first step to fitting the oscillation modes and extracting their frequencies is to correctly identify the modes present. Fortunately, the $\ell=1$ mixed modes follow a frequency pattern that arises from coupling of the p-modes in the envelope, which have approximately equal spacing in frequency $(\Delta \nu)$, to gmodes in the core, which are approximately equally spaced in period $(\Delta \Pi)$. This pattern is well described by the asymptotic relation for mixed modes (Mosser et al. 2012a). We calculated the asymptotic mixed mode frequencies by fitting this relation to several of the highest-amplitude $\ell=1$ modes. From these calculations, nearby peaks could be associated with $\ell=1$ mixed modes. In this way we have been able to identify both of the $m= \pm 1$ components for 21 out of $27 \ell=1$ mixed modes between 200 and $320 \mu \mathrm{Hz}$.

Following a strategy that has been implemented in the mode fitting of other Kepler stars (e.g., Appourchaux et al. 2012), three teams performed fits to the identified modes. The mode frequencies from each fit were compared to the mean values, and the fitter that differed least overall was selected to provide the frequency solution. This fitter performed a final fit to the power spectrum to include modes that other fitters had detected but were absent from this fitter's initial solution.

The final fit was made using a Markov chain Monte Carlo (MCMC) method that performs a global fit to the oscillation spectrum, with the modes modeled as Lorentzian profiles (Handberg \& Campante 2011). Each $\ell=1$ triplet was modeled 


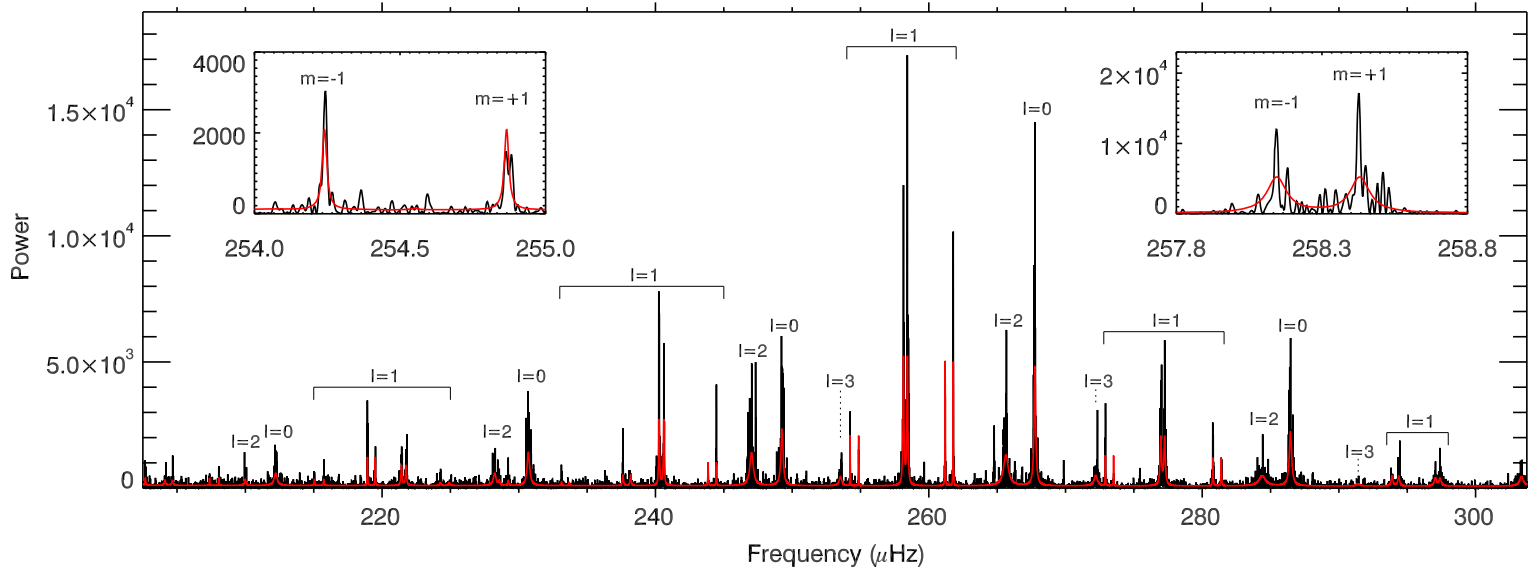

Figure 6. Power spectrum of Kepler-432. The red curve shows the fit to the power spectrum. Insets show a close-up of two rotationally split $\ell=1$ mixed modes. The left inset shows a mixed mode dominated by $g$-mode characteristics, with lower amplitudes, narrower line widths, and a larger rotational splitting relative to the mixed mode on the right, which has strong $p$-mode characteristics.

with the frequency splitting and inclination angle as additional parameters to the usual frequency, height, and width that define a single Lorentzian profile. Owing to the differing sensitivity of the $\ell=1$ mixed modes to rotation at different depths within the star, each $\ell=1$ triplet was fitted with an independent frequency splitting, although a common inclination angle was used. We discuss the rotation of the star further in Section 5.3.

The measured mode frequencies are given in Table 4 . The values of the $\ell=1$ modes are presented as the central frequency of the rotationally split mode profile, which corresponds to the value of the $m=0$ component, along with the value of the rotational splitting between the $m=0$ and $m= \pm 1$ components, $\nu_{s}$. Revised values of $\Delta \nu$ and $\nu_{\max }$ can be obtained from the measured mode frequencies and amplitudes. We find $\Delta \nu=18.59 \pm 0.04 \mu \mathrm{Hz}$ and $\nu_{\max }=266 \pm 3 \mu \mathrm{Hz}$, both of which are in agreement with the values provided by Huber et al. (2013a). Additionally, we measure the underlying $\ell=1$ g-mode period spacing, $\Delta \Pi_{1}$, to be $89.9 \pm 0.3 \mathrm{~s}$, which is consistent with a red giant branch star with a mass below $\sim 1.6 M_{\odot}$ (e.g., Stello et al. 2013).

\subsection{Host Star Inclination}

The line-of-sight inclination of a rotating star can be determined by measuring the relative heights of rotationally split modes (Gizon \& Solanki 2003). A star viewed pole-on produces no visible splitting, while stars viewed with an inclination near $i=45^{\circ}$ would produce a frequency triplet. Figure 6 shows that all dipole modes observed for Kepler-432 are split into doublets, which we interpret as triplets with the central peak missing, indicating a rotation axis nearly perpendicular to the line of sight (inclination $i=90^{\circ}$ ).

To measure the inclination of Kepler-432, we included rotationally split Lorentzian profiles for each of the 21 dipole modes in the global MCMC fit of the power spectrum. Figure 7 shows the posterior distribution of the stellar inclination. The mode of the posterior distribution and $68.3 \%$ highest probability density region is $90.0_{-3.7}^{+0.0} \mathrm{deg}$.

The inclination estimate is based on three important assumptions: that the inclination is the same for p-dominated and g-dominated mixed modes, that there is equipartition of energy between modes with the same $n$ and $\ell$, and that the modes are well resolved.
To test the first assumption, we performed additional fits to individual $\ell=1$ modes using the Python implementaiton of the nested sampling algorithm MultiNest, pyMultiNest (Feroz et al. 2013; Buchner et al. 2014). No significant difference was found between the inclination angle for $\mathrm{p}$-dominated and $\mathrm{g}$ dominated mixed modes. We therefore use the results of our global MCMC fit. Huber et al. (2013b) similarly found no difference between the inclination angle of p-dominated and gdominated mixed modes in Kepler-56. Beck et al. (2014) have shown that these modes actually have slightly different pulsation cavities. They identified asymmetric rotational splittings between $m=0$ and $m= \pm 1$ modes in the red giant KIC 5006817, which results from the modes having varying pand g-mode characteristics. Besides the effect on the rotational splitting, there is also a small impact on mode heights and lifetimes. Beck et al. (2014) further note that the asymmetries are mirrored about the frequency of the uncoupled $p$-modes. This means that the heights of the $m= \pm 1$ components relative to the $m=0$ component will change in opposite directions, so the effect can be mitigated by forcing the $m= \pm 1$ components to have the same height in the fit, as well as by performing a global fit to all modes, as we have done.

Unless mode lifetimes are much shorter than the observing baseline, the Lorentzian profiles of the modes will not be well resolved, and the mode heights will vary. We determined the effect on the measured inclination angle in the manner of Huber et al. (2013b), by investigating the impact on simulated data with similar properties to the frequency spectrum of Kepler432. Taking this effect into account in the determination of our measurement uncertainty, we find a final value of $i=90_{-8}^{+0} \mathrm{deg}$. The asteroseismic analysis therefore shows directly that the spin axis of the host star is in the plane of the sky.

\subsection{Modeling}

Two approaches may be used when performing asteroseismic modeling. The first is the so-called grid-based method, which uses evolutionary tracks that cover a wide range of metallicities and masses and searches for the best-fitting model using $\Delta \nu, \nu_{\max }, T_{\text {eff }}$, and $[\mathrm{Fe} / \mathrm{H}]$ as constraints (e.g., Stello et al. 2009; Basu et al. 2010; Gai et al. 2011; Chaplin et al. 2014). The second is detailed frequency modeling, which uses individual mode frequencies instead of the global 
Table 4

Oscillation Frequencies of Kepler-432

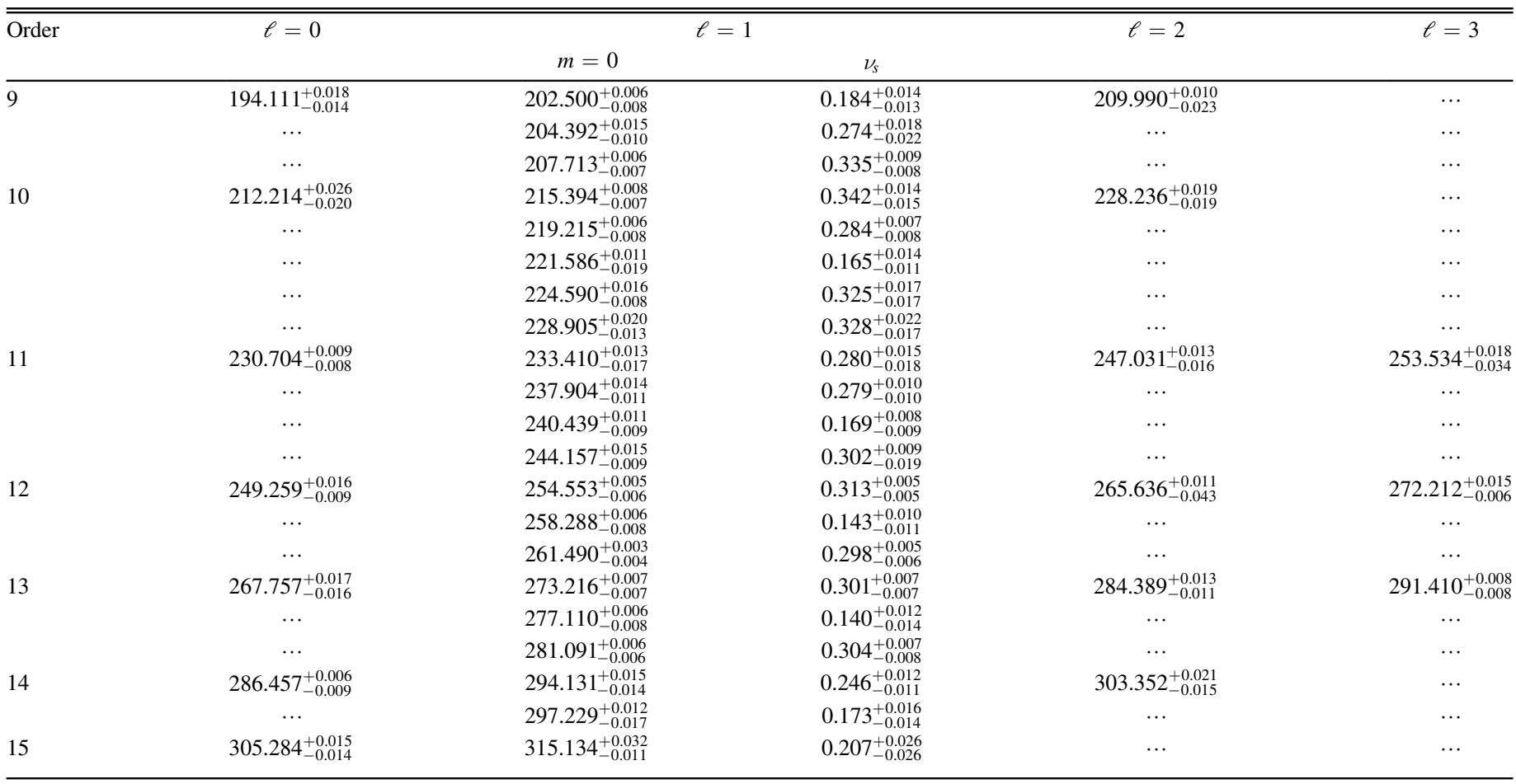

Note. All frequencies are in units of $\mu \mathrm{Hz}$. The $\ell=1$ modes are presented as the central frequency of the rotationally split mode profile $(m=0)$, along with the value of the rotational splitting between the $m=0$ and $m= \pm 1$ components, $\nu_{s}$.

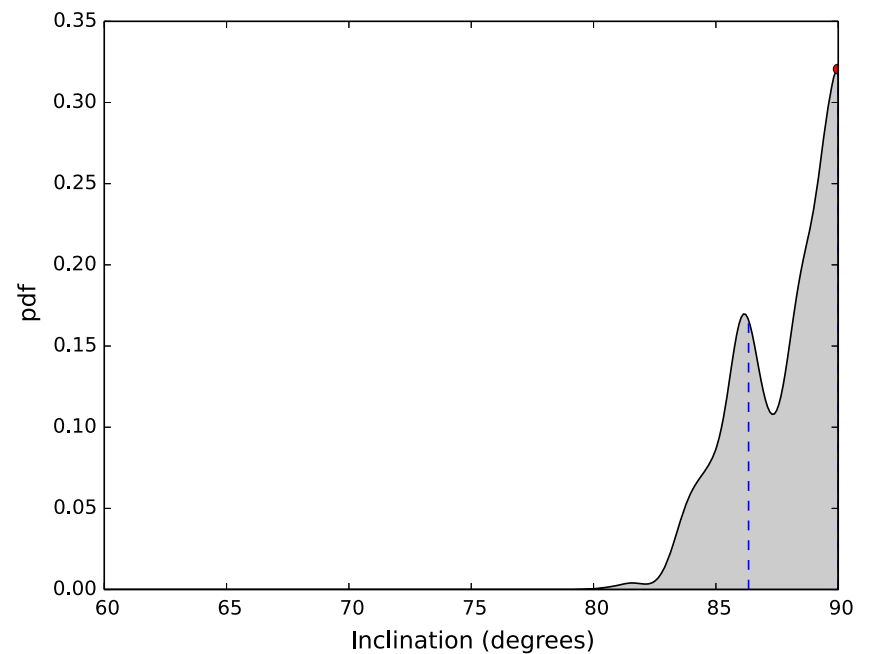

Figure 7. Posterior of the host star line-of-sight inclination derived from the MCMC analysis of the oscillation power spectrum of Kepler-432. The red point indicates the mode of the distribution, and the blue dashed line indicates the limit of the $68 \%$ highest probability density region.

asteroseismic parameters to more precisely determine the bestfitting model (e.g., Metcalfe et al. 2010; Jiang et al. 2011). For comparison, we have modeled Kepler-432 using both approaches.

For the grid-based method we used the Garching Stellar Evolution Code (Weiss \& Schlattl 2008). The detailed parameters of this grid are described by Silva Aguirre et al. (2012), and its coverage has been now extended to stars evolved in the RGB phase. The spectroscopic values of $T_{\text {eff }}$ and $[\mathrm{Fe} / \mathrm{H}]$ found in Section 4.3 and our new asteroseismic measurements of $\Delta \nu$ and $\nu_{\max }$ were used as inputs in a Bayesian scheme as described in Silva Aguirre et al. (2014). Note that while $[\mathrm{Fe} / \mathrm{H}]$ is the model input, our spectroscopic analysis yields an estimate of $[\mathrm{m} / \mathrm{H}]$. We have assumed that the two are equivalent for Kepler-432 (i.e., that the star has a scaled solar composition). If this assumption is invalid, it may introduce a small bias in our results, which are given in Table 3 . The values of mass and radius agree well with those from Huber et al. (2013a), who also used the grid-based method. We note that a comparison of results provided by several grid pipelines by Chaplin et al. (2014) found typical systematic uncertainties of $3.7 \%$ in mass, $1.3 \%$ in radius, and $12 \%$ in age across an ensemble of main-sequence and subgiant stars with spectroscopic constraints on $T_{\text {eff }}$ and $[\mathrm{Fe} / \mathrm{H}]$.

We performed two detailed modeling analyses using separate stellar evolution codes in order to better account for systematic uncertainties. The first analysis modeled the star using the integrated astero extension within MESA (Paxton et al. 2013). After an initial grid search to determine the approximate location of the the global minimum, we found the best-fitting model using the build-in simplex minimization routine, which automatically adjusted the mass, metallicity, and the mixing length parameter. The theoretical frequencies were calculated using GYRE (Townsend \& Teitler 2013) and were corrected for near-surface effects using the power-law correction of Kjeldsen et al. (2008) for radial modes. The non-radial modes in red giants are mixed with g-mode characteristics in the core, so they are less affected by near-surface effects. To account for this, MESA-astero follows Brandão et al. (2011) in scaling the correction term for non-radial modes by $Q_{n, \ell}^{-1}$, where $Q_{n, \ell}$ is the ratio of the inertia of the mode to the inertia of a radial mode at the same frequency. 
The second analysis was performed with the Aarhus Stellar Evolution Code (ASTEC; Christensen-Dalsgaard 2008a), with theoretical frequencies calculated using the Aarhus adiabatic oscillation package (Christensen-Dalsgaard 2008b). The bestfitting model was found in a similar manner as the first analysis, although the mixing length parameter was kept fixed at a value of $\alpha=1.8$.

Figure 8 shows the best-fitting models compared to the observed frequencies in an échelle diagram. Both analyses found an asteroseismic mass and radius of $M=1.32_{-0.07}^{+0.10} M_{\odot}$ and $R=4.06_{-0.08}^{+0.12} R_{\odot}$, but differ in the value of the age, with the best-fitting MESA and ASTEC models having ages of $4.2_{-1.0}^{+0.8}$ and $2.9_{-0.7}^{+0.6} \mathrm{Gyr}$, respectively. Uncertainties were estimated by adopting the fractional uncertainties of the gridbased method, thereby accounting for systematic uncertainties in model input physics and treatment of near-surface effects. The consistency between the detailed model fitting results and grid-based results demonstrates the precise stellar characterization that can be provided by asteroseismology. Throughout the remainder of the paper we adopt the results obtained with the MESA code, though we use an age of $3.5 \mathrm{Gyr}$, which is consistent with both detailed frequency analyses and the age from the grid-based modeling.

\subsection{Distance and Reddening}

The stellar model best fit to the derived stellar properties provides color indices that may be compared against measured values as a consistency check and as a means to determine a photometric distance to the system. Given the physical stellar parameters derived from the asteroseismic and spectroscopic measurements, the PARSEC isochrones (Bressan et al. 2012) predict $J-K_{\mathrm{s}}=0.564$, in good agreement with the measured Two Micron All Sky Survey (2MASS) colors $\left(J-K_{\mathrm{S}}=0.563 \pm 0.028\right)$. While this indicates that the dust extinction along the line of sight is probably low, we attempt to correct for it nonetheless using galactic dust maps. The mean of the reddening values reported by Schlafly \& Finkbeiner (2011) and Schlegel et al. (1998)$E(B-V)=0.079 \pm 0.008$ - is indeed low, implying extinction in the infrared of $\mathrm{A}_{J}=0.070, \mathrm{~A}_{H}=0.045$, and $\mathrm{A}_{K_{s}}=0.028$. Applying these corrections, the observed 2MASS index becomes $J-K_{\mathrm{s}}=0.521$, now slightly inconsistent at the $1 \sigma$ level with the PARSEC model colors. We note that if only a small fraction of the dust column lies between us and Kepler-432, it would lead to a slight overcorrection of the magnitudes and distance. The distances derived in the two cases (using $J, H$, and $K_{s}$ magnitudes) are $878 \pm 9 \mathrm{pc}$ (no extinction) and $859 \pm 13 \mathrm{pc}$ (entire column of extinction). Because the colors agree more closely without an extinction correction, it is tempting to conclude that only a small fraction of the extinction in the direction of Kepler-432 actually lies between us and the star. However, in the direction to the star (i.e., out of the galactic plane), it seems unlikely that a significant column of absorbers would lie beyond $\sim 1 \mathrm{kpc}$. In reality, the model magnitudes are probably not a perfect match for the star and the appropriate reddening for this star is probably between 0 and that implied by the full column (0.079). The derived distance does not depend strongly on the value we adopt for reddening, and we choose to use the mean of the two distance estimates and slightly inflate the errors: $d=870 \pm 20$ pc.

\section{ORBITAL SOLUTION}

After recognition of the signature of the non-transiting planet in the Kepler-432 RVs (the outer planet was identified both visually and via periodogram analysis), they were fit with two Keplerian orbits using an MCMC algorithm with the Metropolis-Hastings rule (Metropolis et al. 1953; Hastings 1970) and a Gibbs sampler (a review of which can be found in Casella \& George 1992). Twelve parameters were included in the fit: for each planet, the times of inferior conjunction $T_{0}$, orbital periods $P$, radial-velocity semi-amplitudes $K$, and the orthogonal quantities $\sqrt{e} \sin \omega$ and $\sqrt{e} \cos \omega$, where $e$ is orbital eccentricity and $\omega$ is the longitude of periastron; the systemic velocity, $\gamma_{\text {rel }}$, in the arbitrary zero point of the TRES relative RV data set; and the FIES RV offset,

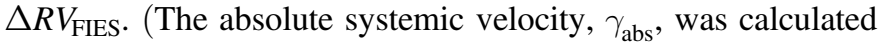
based on $\gamma_{\text {rel }}$ and the offset between relative and absolute RVs, which are discussed in Section 4.) We applied Gaussian priors on $T_{0, \mathrm{~b}}$ and $P_{\mathrm{b}}$ based on the results of the light-curve fitting.

We ran a chain with $1.01 \times 10^{7}$ steps, treating the first $10^{5}$ realizations as burn-in and thinning the chain by saving every 10th entry, for a final chain length of $10^{6}$. The marginalized posterior distributions are shown in Figure 9. It is apparent that while the parameters of the inner planet are very well constrained, there is a high-eccentricity tail of solutions for the outer planet that cannot be ruled out by RVs alone (we investigate this further using $\mathrm{N}$-body simulations in Section 8 ). Because several of the posteriors are non-Gaussian, we cannot simply adopt the median and central $68.3 \%$ confidence interval as our best-fit parameters and $1 \sigma$ errors as we normally might. Instead, we adopt best-fit parameters from the mode of each distribution, which we identify from the peak of the probability density function (PDF). We generate the PDFs using a Gaussian kernel density estimator with bandwidths for each parameter chosen according to Silverman's rule. We assign errors from the region that encloses $68.3 \%$ of the PDF, and for which the bounding values have identical probability densities. That is, we require the $\pm 1 \sigma$ values to have equal likelihoods. The resulting orbital solution using these parameters has velocity residuals larger than expected from the nominal RV uncertainties. We attribute this to some combination of astrophysical jitter (e.g., stellar activity or additional undetected planets) and imperfect treatment of the various noise sources described in Section 4. An analysis of the residuals does not reveal any significant periodicity, but given our measurement precision, we would not expect to detect any additional planets unless they were also massive gas giants, or orbiting at very small separations. To account for the observed velocity residuals, we re-run our MCMC with the inclusion of an additional RV jitter term. Tuning this until $\chi^{2}$ is equal to the number of degrees of freedom, we find that an additional $20 \mathrm{~m} \mathrm{~s}^{-1}$ jitter is required. While part of this jitter may be due to instrumental effects, we do not include separate jitter terms for the TRES and FIES RVs because the FIES data set is not rich enough to reliably determine the observed scatter. We report the best-fit orbital and physical planetary parameters in Table 5, which also includes the set of parameters additionally constrained by dynamical stability simulations, as described in the $N$-body analysis of Section 8 . The corresponding orbital solution is shown in Figure 10. 


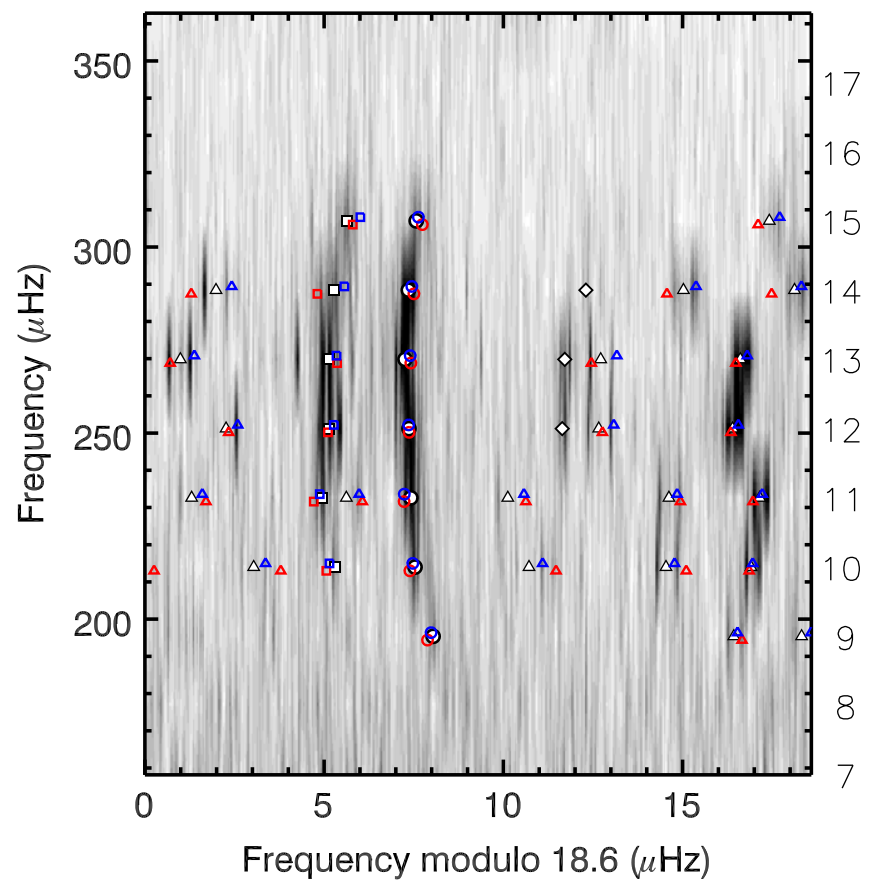

Figure 8. Échelle diagram of Kepler-432 showing observed frequencies in white. Modes are identified as $\ell=0$ (circles), $\ell=1$ (triangles), $\ell=2$ (squares), and $\ell=3$ (diamonds). Frequencies of the best-fitting MESA and ASTEC models are indicated by the red and blue open symbols, respectively. For reference, a gray-scale map of the power spectrum is shown in the background. Numbers to the right of the plot indicate the radial order of the $\ell=0$ modes.

\section{FALSE POSITIVE SCENARIOS}

An apparent planetary signal (transit or RV) can sometimes be caused by astrophysical false positives. We consider several scenarios in which one of the Kepler-432 planetary signals is caused by something other than a planet, and we run a number of tests to rule these out.

For an object with a deep transit, such as Kepler-432b, one may worry that the orbiting object is actually a small star, or that the signal is caused by a blend with an eclipsing binary system. Sliski \& Kipping (2014) noted that there are very few planets orbiting evolved stars with periods shorter than 100 days (Kepler-432b would be somewhat of an outlier) and also found via AP that either the transit signal must be caused by a blend or it must have significant eccentricity $(e>0.488)$. Without additional evidence, this would be cause for concern, but our radial velocity curve demonstrates that the transiting object is indeed orbiting the target star, its mass is planetary, and, consistent with the prediction of Sliski \& Kipping (2014), its eccentricity is 0.5134 .

If a planet does not transit, as is the case for Kepler-432c, determining the authenticity of the planetary signal is less straightforward. An apparent radial-velocity orbit can be induced by a genuine planet, spots rotating on the stellar surface (Queloz et al. 2001), or a blended stellar binary (Mandushev et al. 2005). Both of these false positive scenarios should manifest themselves in the shapes of the stellar spectral lines. That is, spots with enough contrast with the photosphere to induce apparent RV variations will also deform the line profiles, as should blended binaries bright enough to influence the derived RVs. A standard prescription for characterizing the shape of a line is to measure the relative velocity at its top and bottom; this difference is referred to as a line bisector span (see, e.g., Torres et al. 2005). To test against the scenarios described, we computed the line bisector spans for the TRES spectra. We do find a possible correlation with the RVs of the outer planet, having a Spearman's rank correlation value of -0.21 and a significance of $94 \%$. While this is a potential concern and we cannot conclusively demonstrate the planetary nature of the 407 day signal, we find it to be the most likely interpretation. In the following paragraphs, we explain why other interpretations are unlikely.

With the discovery of a close stellar companion (see Section 3.2), it is reasonable to ask how that might affect interpretation of the outer planetary signal. That is, if the stellar companion is itself a binary with a period of $\sim 400$ days, could it cause the RV signal we observe? The answer is unequivocally no, as the companion is far too faint in the optical compared to the primary star $(\Delta V \sim 7)$ to contribute any significant light to the spectrum, let alone induce a

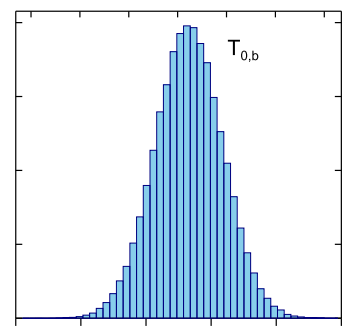

687.0280687 .0307687 .0334687 .0360

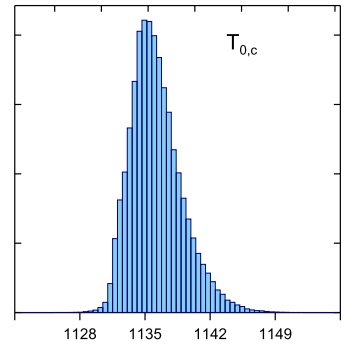

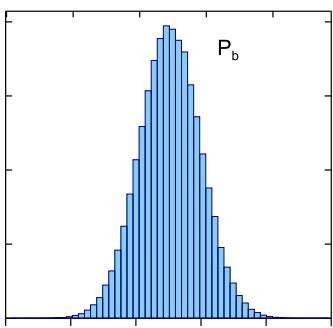

$52.5008 \quad 52.501052 .501252 .5014$

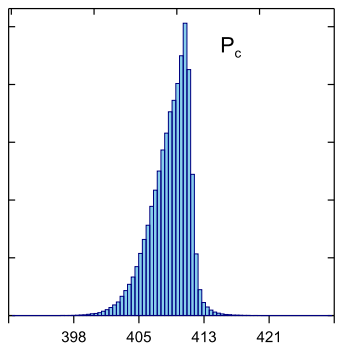

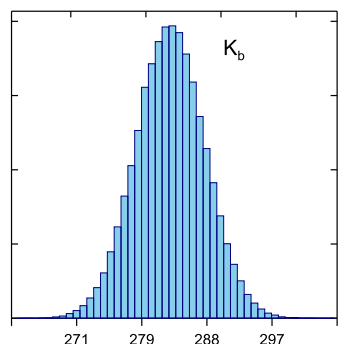

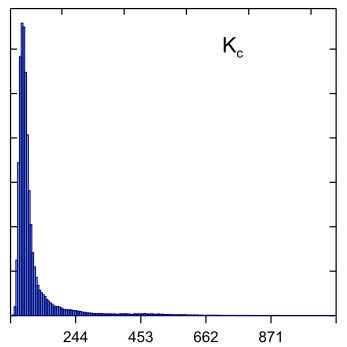

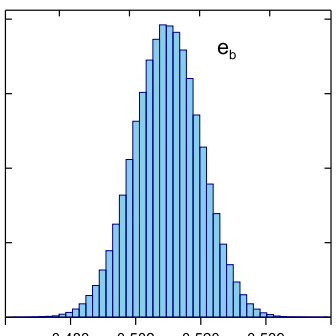

$\begin{array}{llll}0.483 & 0.502 & 0.520 & 0.539\end{array}$

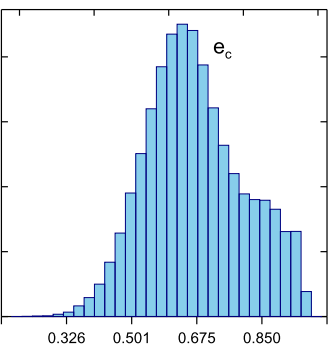

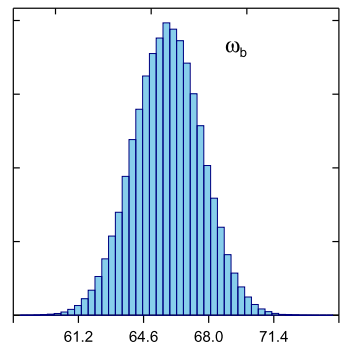

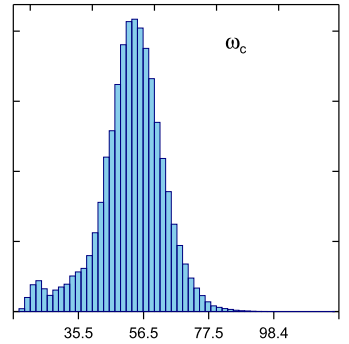

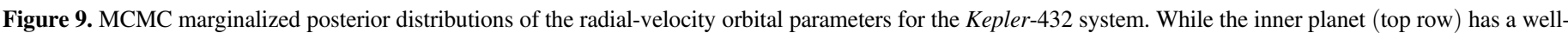

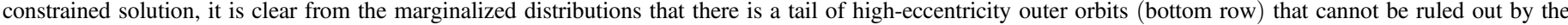
current RV data. This tail can also be seen in the joint mass-eccentricity posterior shown in the stability analysis of Figure 11. 
Table 5

Kepler-432 Planetary Properties

\begin{tabular}{lrr}
\hline \hline Parameter & Without $N$-Body & With $N$-Body \\
\hline Inner Planet & & \\
\hline$T_{0, \mathrm{~b}}(\mathrm{BJD})$ & $2455949.5374_{-0.0016}^{+0.0018}$ & $2455949.5374_{-0.0012}^{+0.0011}$ \\
$P_{\mathrm{b}}($ days $)$ & $52.501134_{-0.000107}^{+0.00070}$ & $52.501129_{-0.000053}^{+0.000067}$ \\
$K_{\mathrm{b}}\left(\mathrm{ms}^{-1}\right)$ & $285.9_{-4.7}^{+4.1}$ & $286.8_{-4.0}^{+4.7}$ \\
$\sqrt{e_{\mathrm{b}}} \cos \omega_{\mathrm{b}}$ & $0.294_{-0.015}^{+0.022}$ & $0.311_{-0.016}^{+0.018}$ \\
$\sqrt{e_{\mathrm{b}}} \sin \omega_{\mathrm{b}}$ & $0.6482_{-0.0094}^{+0.0134}$ & $0.645_{-0.011}^{+0.012}$ \\
$e_{\mathrm{b}}$ & $0.5121_{-0.0107}^{+0.0084}$ & $0.5134_{-0.0089}^{+0.0098}$ \\
$\omega_{\mathrm{b}}(\mathrm{deg})$ & $65.6_{-1.8}^{+1.5}$ & $64.1_{-1.5}^{+1.6}$ \\
$i_{b}(\mathrm{deg})$ & $88.17_{-0.33}^{+0.61}$ & $88.17_{-0.33}^{+0.61}$ \\
$M_{\mathrm{b}}\left(M_{\mathrm{Jup}}\right)$ & $5.41_{-0.19}^{+0.30}$ & $5.41_{-0.18}^{+0.32}$ \\
$R_{\mathrm{b}}\left(R_{\mathrm{Jup}}\right)$ & $1.145_{-0.039}^{+0.036}$ & $1.145_{-0.039}^{+0.036}$ \\
$\rho_{\mathrm{b}}\left(\mathrm{g} \mathrm{cm}{ }^{-3}\right)$ & $4.46_{-0.29}^{+0.36}$ & $4.46_{-0.29}^{+0.37}$ \\
$a_{\mathrm{b}}(\mathrm{AU})$ & $0.301_{-0.011}^{+0.016}$ & $0.301_{-0.011}^{+0.016}$ \\
$\left\langle F_{\mathrm{b}}\right\rangle\left(\left\langle F_{\oplus}\right\rangle\right)^{\mathrm{a}}$ & $118 \pm 10$ & $118 \pm 10$ \\
\hline
\end{tabular}

Outer Planet

\begin{tabular}{lrr}
\hline$T_{0, \mathrm{c}}(\mathrm{BJD})$ & $2456134.9_{-2.1}^{+3.0}$ & $2456139.3_{-2.9}^{+3.6}$ \\
$P_{\mathrm{c}}($ days $)$ & $411.0_{-3.2}^{+0.9}$ & $406.2_{-2.5}^{+3.9}$ \\
$K_{\mathrm{c}}\left(\mathrm{ms}^{-1}\right)$ & $73_{-15}^{+25}$ & $62.1_{-5.8}^{+6.1}$ \\
$\sqrt{e_{\mathrm{c}}} \cos \omega_{\mathrm{c}}$ & $0.47_{-0.14}^{+0.12}$ & $0.336_{-0.076}^{+0.115}$ \\
$\sqrt{e_{\mathrm{c}}} \sin \omega_{\mathrm{c}}$ & $0.648_{-0.073}^{+0.072}$ & $0.602_{-0.071}^{+0.048}$ \\
$e_{\mathrm{c}}$ & $0.64_{-0.13}^{+0.14}$ & $0.498_{-0.059}^{+0.029}$ \\
$\omega_{\mathrm{c}}(\operatorname{deg})$ & $53.2_{-9.5}^{+8.7}$ & $60.8_{-11.2}^{+7.0}$ \\
$M_{\mathrm{c}} \sin i_{\mathrm{c}}\left(M_{\mathrm{Jup}}\right)$ & $2.63_{-0.35}^{+0.43}$ & $2.43_{-0.24}^{+0.22}$ \\
$a_{\mathrm{c}} \sin i_{\mathrm{c}}(\mathrm{AU})$ & $1.188_{-0.042}^{+0.062}$ & $1.178_{-0.042}^{+0.063}$ \\
$\left\langle F_{\mathrm{c}}\right\rangle\left(\left\langle F_{\oplus}\right\rangle\right)^{\mathrm{a}}$ & $8.5_{-1.2}^{+2.1}$ & $7.7_{-0.8}^{+0.7}$ \\
\hline
\end{tabular}

Other Parameters

\begin{tabular}{lrr}
\hline$\gamma_{\text {rel }}\left(\mathrm{ms}^{-1}\right)$ & $313.4_{-4.5}^{+5.1}$ & $306.7_{-2.7}^{+2.6}$ \\
$\gamma_{\mathrm{abs}}\left(\mathrm{kms}^{-1}\right)$ & $-35.22 \pm 0.19$ & $-35.22 \pm 0.19$ \\
$\Delta R V_{\mathrm{FIES}}\left(\mathrm{ms}^{-1}\right)$ & $-493.5_{-10.4}^{+8.4}$ & $-490.5_{-7.7}^{+8.6}$ \\
$\mathrm{RV} \mathrm{Jitter}\left(\mathrm{ms}^{-1}\right)$ & 20 & 20 \\
\hline
\end{tabular}

Note. The first set of parameters comes from the the photometric, radialvelocity, and asteroseismic analyses, and the second set incorporates additional constraints from the stability analysis of our $N$-body simulations. We adopt the properties derived with constraints from the $N$-body simulations.

${ }^{\mathrm{a}}$ The time-averaged incident flux, $\langle F\rangle=\sigma T_{\mathrm{eff}}^{4}\left(\frac{R_{\star}}{a}\right)^{2}\left(\frac{1}{1-e^{2}}\right)^{1 / 2}$.

variation of $\sim 100 \mathrm{~m} \mathrm{~s}^{-1}$ or affect the bisector spans. If there is also a brighter visual companion inside the resolution limit of our high-resolution images $\left(\sim 00^{\prime \prime} 05 \simeq 45 \mathrm{AU}\right.$ projected separation), it could be a binary with a 400 day period responsible for the $\mathrm{RV}$ and bisector span variations on that timescale. However, such a close physically bound binary may pose problems for formation of the 52.5 day planet, and the a priori likelihood of a background star bright enough to cause the observed variations within $0{ }^{\prime \prime} 05$ is extremely low; a TRILEGAL simulation suggests $\sim 5 \times 10^{-6}$ background sources should be expected, and only a small fraction of those would be expected to host a binary with the correct systemic velocity.

To rule out spot-induced velocity variation, we examine the Kepler light-curve for evidence of spot activity. From the measured $v \sin i_{\star}, i_{\star}$, and $R_{\star}$, the stellar rotation period is $77 \pm 14$ days. Not only is this inconsistent with the observed outer orbital period (407 days), but we detect no significant photometric signal near either of these periods. For Kepler-432 ( $v \sin i_{\star}=2.7 \mathrm{~km} \mathrm{~s}^{-1}$ ), a spot must cover $\sim 4 \%-5 \%$ of the stellar surface to induce the observed RV amplitude (Saar \& Donahue 1997), and such a spot would have been apparent in the high-precision Kepler light-curve.

As we have shown in Section 5, Kepler-432 exhibits strong oscillations, so one may wonder whether these could induce the observed RV signal for the outer planet. Oscillations on a 400 day timescale are intrinsically unlikely, as there is no known driving mechanism that could cause them in giant stars; the well-known stochastically driven oscillations are confined to much higher frequencies. Furthermore, if there were such a mechanism, a mode with $\sim 50 \mathrm{~m} \mathrm{~s}^{-1}$ velocity semi-amplitude should cause a photometric variation of $\sim 1.3 \mathrm{mmag}$ (see Kjeldsen \& Bedding 1995), which is clearly ruled out by the Kepler data.

Upon examining all of the evidence available, we conclude that both detected orbits are caused by bona fide planets orbiting the primary star.

\section{ORBITAL STABILITY ANALYSIS}

\subsection{Methodology}

Following the Keplerian MCMC fitting procedure described in Section 6, we wish to understand whether these posterior solutions are dynamically stable-i.e., whether they describe realistic systems that could survive to the $3.5 \mathrm{Gyr}$ age of the system. When presenting our results in the sections that follow, we use the planet-planet separation as an easily visualized proxy for stability: given the semi-major axes of the two planets $\sim 0.3$ and $\sim 1.2$ AU-separations greater than $\sim 10$ AU are clear indications that the system has suffered an instability and the planets subsequently scattered. If some solutions do prove to be unstable, it will lead to further constraints on the orbital elements and thus the planetary masses. We perform integrations of both coplanar and inclined systems. We first explore the less computationally expensive coplanar case to understand the behavior of the system, and then extend the simulations to include inclination in the outer orbit, which has the additional potential to constrain the mutual inclination of the planets.

To understand this stability, we utilize the integration algorithm described in Payne et al. (2013). This algorithm uses a symplectic method in Jacobi coordinates, making it both accurate and rapid for systems with arbitrary planet-to-star mass ratios. It uses calculations of the tangent equations to evaluate the Lyapunov exponents for the system, providing a detailed insight into whether the system is stable (up to the length of the simulation examined) or exhibits chaos (and hence instability).

For the coplanar systems, we take the ensemble of $10^{6}$ solutions generated in Section 6 (which form the basis of the reported elements in column 2 of Table 5) and convert these to Cartesian coordinates, assuming that the system is coplanar and edge-on $\left(90^{\circ}\right)$, and hence both planets have their minimum masses. We then evolve the systems forward for a fixed period of time (more detail supplied in Section 8.2 below) and examine some critical diagnostics for the system (e.g., the Lyapunov time, and the planet-planet separation) to 

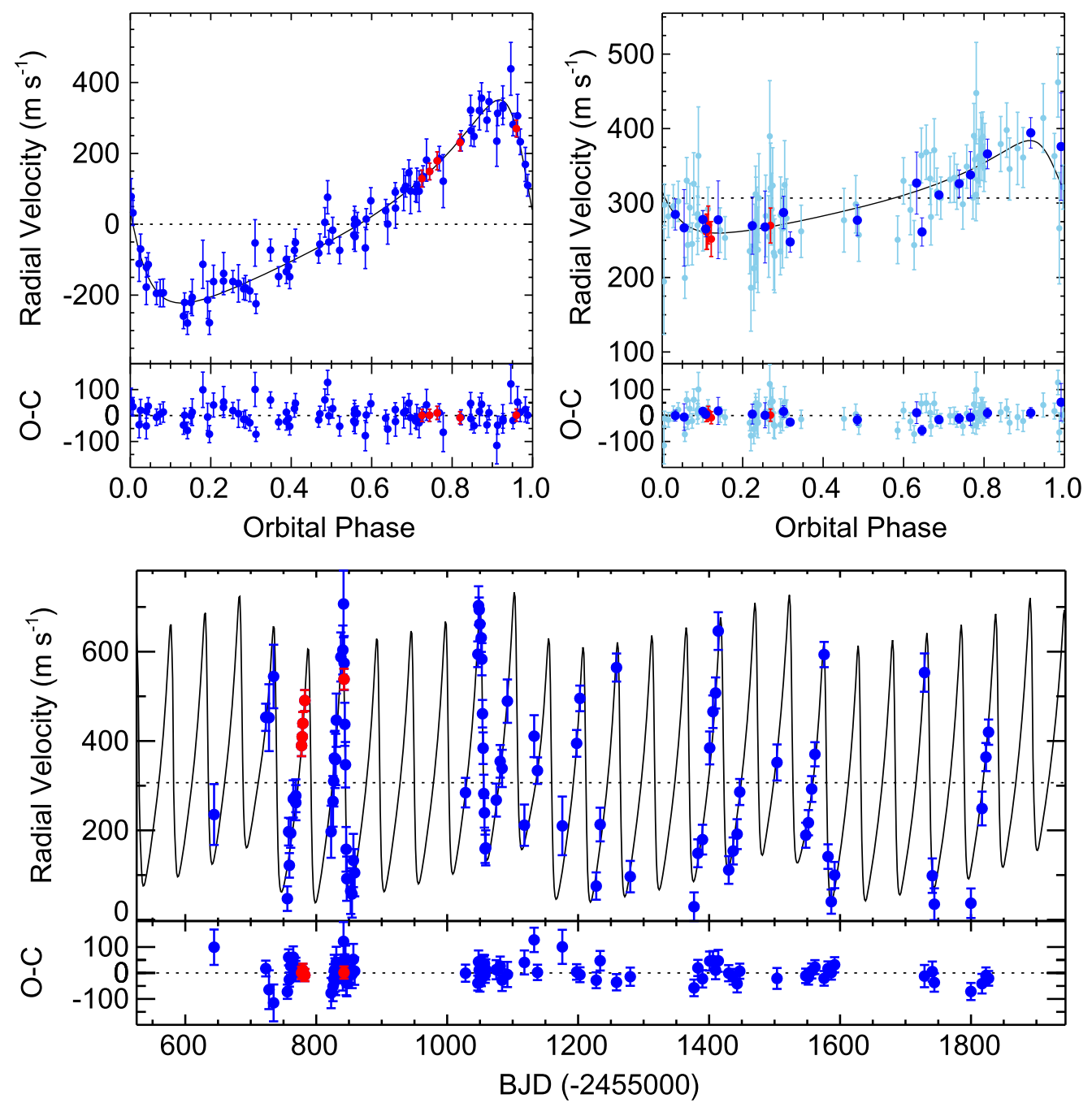

Figure 10. Individual components of the orbital fit corresponding to planets b (top left) and c (top right), and the combined RV time series (bottom). TRES RVs are plotted in blue and FIES RVs are plotted in red. For planet c, we plot the individual RVs in light blue and the binned data in dark blue. Velocity residuals are plotted in the corresponding lower panels. The plotted error bars include both the internal errors and the $20 \mathrm{~m} \mathrm{~s}^{-1}$ jitter that was added to the uncertainties during the fitting process.

understand whether the system remains stable, or whether some significant instability has become apparent.

For the inclined systems, we assume that the inner (transiting) planet is edge-on and hence retains its measured mass. However, the outer planet is assigned an inclination that is drawn randomly from a uniform distribution $0^{\circ}<i_{\mathrm{c}}<90^{\circ}$, and its mass is scaled by a factor $1 / \sin i_{\mathrm{c}}$. As such, the outer planet can have a mass that is significantly above the minimum values used in the coplanar case. The longitude of ascending node for the outer planet is drawn randomly from a uniform distribution between 0 and $2 \pi$. We then proceed as in the coplanar case, integrating the systems forward in time to understand whether the initial conditions chosen can give rise to long-term stable systems.

Rauch \& Holman (1999) demonstrated that 20 time steps per innermost orbit is sufficient to ensure numerical stability in symplectic integrations. As the inner planet has a period of $\sim 50$ days, we use a time step of 1 day in all of our simulations, ensuring that our integrations will comfortably maintain the desired energy conservation and hence numerical accuracy.

\subsection{Coplanar Stability}

We begin by taking a random selection of $10^{5}$ of the $10^{6}$ solutions from Section 6 and integrating them for a period of $10^{4} \mathrm{yr}\left(3 \times 10^{6}\right.$ time steps). While this is not a particularly long integration period compared with the period of the planets ( $\sim 50$ and $\sim 400$ days), we demonstrate that even during this relatively short integration, approximately half of the systems become unstable. Tellingly, the unstable systems all tend to be the systems in which the outer planet has particularly high eccentricity. We illustrate this in Figure 11, where we plot the mass-eccentricity plane for the outer planet $\left(m_{\mathrm{c}}, e_{\mathrm{c}}\right)$ and plot the separation between the two planets in the system at $t=10^{4} \mathrm{yr}$. As described above, separations greater than $\sim 10$ AU indicate that the system has suffered an instability. This initial simulation clearly demonstrates that at $t=10^{4} \mathrm{yr}$ essentially all systems with $e_{\mathrm{c}}>0.8$ are unstable, all those with $e_{\mathrm{c}}<0.45$ are stable, and those with $0.45<e_{\mathrm{c}}<0.8$ are "mixed," with some being stable and some being unstable.

Given this promising demonstration that the dynamical integrations can restrict the set of solutions, we go on to integrate the systems for increasingly longer periods of time. 

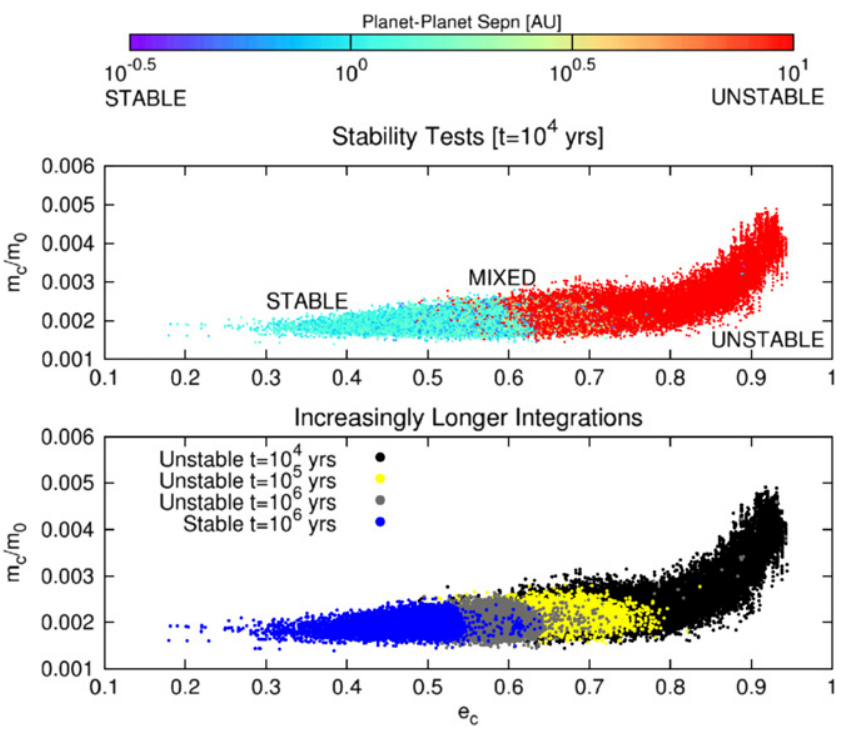

Figure 11. Top: in the mass-eccentricity plane for the outer planet $\left(m_{c}, e_{c}\right)$, we plot the separation between the two planets in the system at $t=10^{4} \mathrm{yr}$ using the color scheme at the top of the plot. A separation greater than $\sim 10 \mathrm{AU}$ is an indication that the system has suffered an instability and the planets subsequently scattered. Systems with eccentricity $e_{c} \gtrsim 0.75$ are clearly unstable, while those with $e_{c} \lesssim 0.55$ are clearly stable for $t=10^{4}$ yr. Bottom: in the $\left(m_{c}, e_{c}\right)$ plane, we plot the systems which are unstable at $10^{4} \mathrm{yr}$ in black, $10^{5} \mathrm{yr}$ in yellow, and $10^{6} \mathrm{yr}$ in gray, and those that remain stable at $10^{6} \mathrm{yr}$ in blue. The systems that are long-term stable in these coplanar simulations occupy a region of parameter space confined to eccentricities $e_{c} \lesssim 0.55$.

To save on integration time/cost, we only select the stable solutions from the previous step and then extend the integration time by an order of magnitude, perform a stability analysis, and repeat. By this method, the $10^{5}$ systems at $t=0$ are reduced to $\sim 4.4 \times 10^{4}$ stable systems at $10^{4} \mathrm{yr}, \sim 2.5 \times 10^{4}$ stable systems at $10^{5} \mathrm{yr}$, and $\sim 9.4 \times 10^{3}$ stable systems at $10^{6} \mathrm{yr}$. We illustrate in Figure 11 the successive restriction of the parameter space in the mass-eccentricity plane for the outer planet $\left(m_{\mathrm{c}}, e_{\mathrm{c}}\right)$ as the integration timescales increase. We find that the long-term stable systems occupy a significantly smaller region of parameter space in the $m_{\mathrm{c}}, e_{\mathrm{c}}$ plane. In particular, we see that the eccentricity of the outer planet is restricted to $e_{\mathrm{c}} \lesssim 0.55$.

Using the $\sim 9.4 \times 10^{3}$ systems that remained stable at $10^{6} \mathrm{yr}$, we use the same method as in Section 6 to determine a new set of best-fit orbital paramters. That is, we use a Gaussian kernel density estimator to smooth the posterior distribution and select the mode (i.e., the value with the largest probability density). The errors correspond to values with equal probability density that enclose the mode and $68.3 \%$ of the PDF. The most significant changes in the best-fit parameters occurred for the outer planet, most notably for eccentricity and velocity semiamplitude, resulting in smaller and more symmetric error bars and a revision in the best-fit minimum mass for planet $\mathrm{c}$.

\subsection{Inclined-system Stability}

In a manner similar to the coplanar analysis of Section 8.2, we begin by taking all $10^{6}$ solutions from Section 6 and integrating them for a period of $10^{4} \mathrm{yr}\left(3 \times 10^{6}\right.$ time steps $)$. However, in these inclined system integrations, the outer planet has an inclination that is not edge-on, and hence a mass for the outer planet that is inflated compared to its minimum edge-on value, as described in Section 8.1.

A much larger fraction of the inclined systems become unstable in the first $10^{4} \mathrm{yr}$, and we find that the $N=10^{6}$ systems at $t=0$ are reduced to $N \sim 1.5 \times 10^{5}$ stable systems at $10^{4} \mathrm{yr}$. Integrating these stable systems while keeping the pre-assigned inclinations for the outer planet (i.e., we do not rerandomize the inclinations), we integrate this subset on to $10^{5} \mathrm{yr}$ and find that the number of stable systems subsequently reduces to $N \sim 10^{4}$. We plot these stability results as a function of mass and inclination of the outer planet in Figure 12.

We find that the vast majority of the stable solutions are (somewhat unsuprisingly) restricted to the range of parameter space with relative inclinations $\lesssim 70^{\circ}$ (i.e., $i_{\mathrm{c}} \gtrsim 20^{\circ}$ in Figure 12). There is a small population with significantly higher eccentricities and relative inclinations that remains stable at $t=10^{5} \mathrm{yr}$, and it is possible that these systems exhibit strong Kozai oscillations, but their long-term $\left(>10^{5} \mathrm{yr}\right)$ behavior has not been investigated. We emphasize that the inclinations and longitudes of the ascending node were assigned randomly, and hence it is possible that specifically chosen orbital alignments could allow for enhanced stability in certain cases. Unfortunately, because some systems remain stable even for highly inclined outer orbits $\left(i_{c} \sim 0^{\circ}\right)$, we are unable to strongly constrain the mutual inclination of the planetary orbits.

We also note that the presence of a distant stellar companion (as detected in our AO images) or additional, as yet undetected, planets (as may be suggested by the excess RV scatter) could influence the long-term stability of the systems simulated herein. The evolution of the star on the red giant branch is also likely to affect the orbital evolution (especially for the inner planet; see Section 9), but it is not important over the $10^{6} \mathrm{yr}$ timescales of these simulations. Simulating the effect of poorly characterized or hypothetical orbits, or the interactions between expanding stars and their planets, is beyond the scope of the current paper, and instead we simply remind the reader of these complications. We present planetary properties derived with and without constraints from our simulations (Table 5) so that the cautious reader may choose to adopt the more conservative (and poorly determined) parameters of the outer orbit in any subsequent analysis.

\section{DISCUSSION}

The properties of the star and planets of Kepler-432 are unusual in several ways among the known exoplanets, which makes it a valuable system to study in detail. For example, it is a planetary system around an intermediate-mass star (and an evolved star), it hosts a planet of intermediate period, and it hosts at least one very massive transiting planet. Close examination of the system may provide insight into the processes of planet formation and orbital evolution in such regimes. Kepler-432 is also the first planet orbiting a giant star to have its eccentricity independently determined by RVs and photometry, which helps address a concern that granulation noise in giants can inhibit such photometric measurements.

\subsection{Comparing the Eccentricity from AP versus $R V s$}

AP provides an independent technique for measuring orbital eccentricities with photometry alone, via the so-called photoeccentric effect (Dawson \& Johnson 2012), and can be used as 


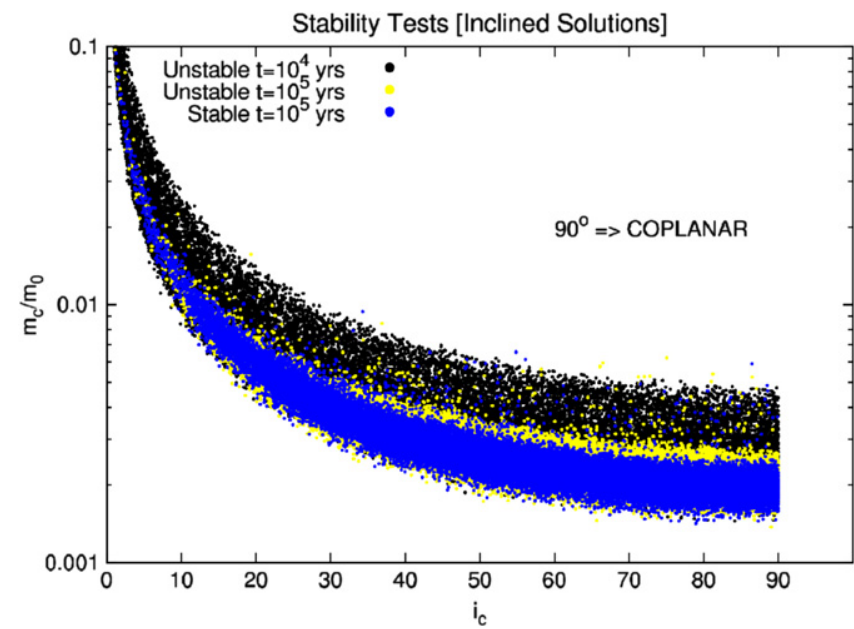

Figure 12. Stability of systems in the mass-orbital inclination plane of the outer planet $\left(m_{\mathrm{c}}, i_{\mathrm{c}}\right)$, with black points going unstable within $10^{4} \mathrm{yr}$ and yellow points within $10^{5} \mathrm{yr}$, and blue points representing stable systems after integrating for $10^{5} \mathrm{yr}$. The results mirror those of the coplanar case-at all inclinations, the low-mass (low-eccentricity) systems remain stable, while the high-mass (high-eccentricity) systems do not survive long integrations. As some systems remain stable even for highly inclined orbits $\left(i_{\mathrm{c}} \sim 0^{\circ}\right)$, we are unable to put strong constraints on the mutual inclination of the planets.

a tool to evaluate the quality of planet candidates (Tingley et al. 2011). It was originally envisioned as a technique for measuring eccentricities (Kipping et al. 2012), but other effects, such as a background blend, can also produce AP effects (Kipping 2014a). Given that we here have an independent radial velocity orbital solution, there is an opportunity to compare the two independent solutions, allowing us to comment on the utility of AP.

Kepler-432b was previously analyzed as part of an ensemble AP analysis by Sliski \& Kipping (2014). In that work, the authors concluded that Kepler-432b displayed a strong AP effect, either because the candidate was a false positive or because it exhibited a strong photoeccentric effect with $e \geqslant 0.488_{-0.051}^{+0.025}$. Our analysis, using slightly more data and a full $\omega$ marginalization, is in excellent agreement with that result, finding $e=0.507_{-0.114}^{+0.039}$ and $\omega=76_{-24}^{+59} \mathrm{deg}$. The AP results may be compared to that from our radial velocity solution, where again we find excellent agreement, since RVs yield $e=0.5134_{-0.0089}^{+0.0098}$ and $\omega=64.1_{-1.5}^{+1.6} \mathrm{deg}$. The results may be visually compared in Figure 13 . We note that this is not the first time AP and RVs have been shown to yield self-consistent results, with Dawson \& Johnson (2012) demonstrating the same for the Sun-like star HD 17156b. However, this is the first time that this agreement has been established for a giant host star. This is particularly salient in light of the work of Sliski \& Kipping (2014), who find that the AP deviations of giant stars are consistently excessively large. The authors proposed that many of the KOIs around giant stars were actually orbiting a different star, with the remaining being eccentric planets around giant stars. Kepler-432b falls into the latter category, consistent with its original ambiguous categorization as being either a false positive or photoeccentric.

The analysis presented here demonstrates that AP can produce accurate results for giant stars. This indicates that the unusually high AP deviations of giant stars observed by Sliski \& Kipping (2014) cannot be solely due to time-correlated noise caused by stellar granulation, which has recently been proposed by Barclay et al. (2014) to explain the discrepancy between AP and radial velocity data for Kepler-91b. However, timecorrelated noise may still be an important factor in giant host stars that are more evolved than Kepler-432 (such as Kepler91), for which granulation becomes more pronounced (Mathur et al. 2011). To further investigate these hypotheses, we advocate for further observations of giant planet-candidate host stars to resolve the source of the AP anomalies.

\subsection{A Benchmark for Compositions of Super-Jupiters}

Kepler-432b has a measured mass, radius, and age, which allows us to investigate its bulk composition. Among transiting planets-i.e., those for which interior modeling is possiblethere are only 11 more massive than $4.5 M_{\text {Jup }}$. We also point out the gap in the center of the plot in Figure 14; no planets with masses between 4 and $7.25 M_{\text {Jup }}$ also have measured radii, so Kepler-432b immediately becomes a valuable data point to modelers. Moreover, most of the super-Jupiters are highly irradiated, which further complicates modeling and interpretation of planetary structure. Kepler-432b receives only about $20 \%$ of the insolation of a 3 day hot Jupiter orbiting a Sun-like star. Because of this, it may prove to be an important benchmark for planetary interior models-for example, as a means of checking the accuracy of models of super-Jupiters without the complication of high levels of incident flux. In Figure 14, we compare the mass and radius of Kepler-432b to age- and insolation-appropriate planetary models (Fortney et al. 2007) of varying core mass. We interpolate the models to a planet with the age of Kepler-432 $(3.5 \mathrm{Gyr})$ in a circular orbit of $0.092 \mathrm{AU}$ around a Sun-like star (the insolation of which is identical to the time-averaged insolation of Kepler-432b on its more distant eccentric orbit around a more luminous star). The radius is apparently slightly inflated but is somewhat consistent $(1 \sigma)$ with that of a planet lacking a core of heavy elements. This is not an iron-clad result, though; the radius even agrees with the prediction for a planet with a $50 M_{\oplus}$ core to within $1.5 \sigma$. Nevertheless, we interpret this as evidence that Kepler$432 \mathrm{~b}$ most likely has only a small core of heavy elements.

\subsection{Jupiters Do (Briefly) Orbit Giants within 0.5 AU}

As discussed previously, we do not know of many transiting giant planets orbiting giant stars. This is not because giant planets orbiting the progenitors to giant stars are rare; due in part to survey strategies, giant stars are, on average, more massive than the known main-sequence planet hosts, and massive stars seem to be more likely to harbor massive planets. However, because very few giant stars host planets inside $1 \mathrm{AU}$, the a priori probability of a transit is low for these systems. In fact, there are only two other giant planets with a measured mass $\left(M_{p}>0.5 M_{\text {Jup }}\right)$ transiting a giant star $\left(\log g_{\star}<3.9\right)-$ Kepler-56c (Huber et al. 2013b), and the soon-to-be-swallowed hot Jupiter Kepler-91b (Barclay et al. 2014; Lillo-Box et al. 2014b, 2014a). This makes Kepler-432b important in at least two respects: it is a rare fully characterized giant planet transiting a giant star, and it is a short-period outlier among giant planets orbiting giant stars (see Figure 15). Kepler-432b thus gives us a new lens through which to examine the dearth of short- and intermediate-period Jupiters $(<0.5 \mathrm{AU})$ around giant stars. Kepler-432c, on the other hand, appears to be very typical of Jupiters around evolved stars, in both mass and orbital separation. 

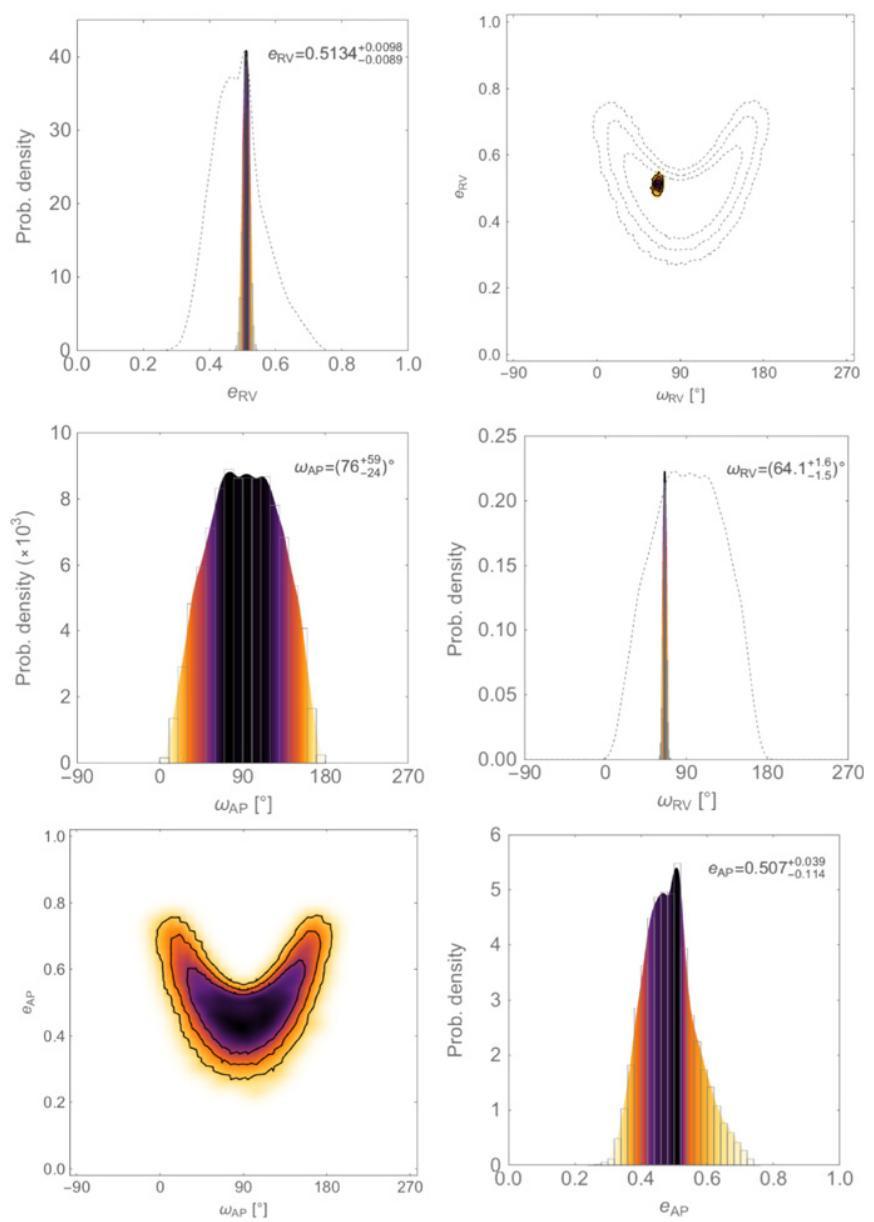

Figure 13. Marginalized and joint posterior distributions of $e$ and $\omega$, as determined by AP and RVs. The three panels in the lower left are from AP; the three in the upper right (the narrow histograms and well-constrained joint distribution) are from RVs, which also show the AP distributions overplotted as thin gray dotted lines for comparison.

After noticing that Kepler-432b sits all alone in the $M_{\mathrm{p}}$ versus $a$ parameter space, it is natural to wonder why, and we suggest two potential explanations, each of which may contribute to this apparent planetary desert. In the first, we consider that Kepler-432b may simply be a member of the tail of the period distribution of planets around massive and intermediate-mass main-sequence stars. That is, perhaps massive main-sequence stars simply harbor very few planets with separations less than $1 \mathrm{AU}$. If this occurrence rate is a smooth function of stellar mass, then because Kepler-432 would most accurately be called intermediate mass, it might not be so surprising that it harbors a planet with a separation of $0.3 \mathrm{AU}$ while its more massive counterparts do not. If this is the case, then as we detect more giant planets orbiting giant stars (and main-sequence stars above the Kraft break), we can expect to find a sparsely populated tail of planets interior to $1 \mathrm{AU}$. This may ultimately prove to be responsible for the observed distribution and would have important implications for giant planet formation and migration around intermediatemass stars in comparison to Sun-like stars, but it cannot be confirmed now. It will require additional planet searches around evolved stars and improvements in detecting longperiod planets orbiting rapidly rotating main-sequence stars.

In the second scenario, we consider that Kepler-432b may be a member of a more numerous group of planets interior to $\sim 0.5$ AU that exist around main-sequence F- and A-type stars but do not survive through the red giant phase when the star expands to a significant fraction of an AU. If this is the case, then Kepler-432b is fated to be swallowed by its star through some combination of expansion of the stellar atmosphere and orbital decay, and we only observe it now because Kepler-432 has only recently begun its ascent up the red giant branch. Suggestively, the resulting system that would include only Kepler-432c would look much more typical of giant stars. In this scenario, planets must begin their orbital decay very soon after the star evolves off of the main sequence, or we would expect to observe many more of them. Assuming, then, that Kepler-432b is close enough to its host to have started its orbital decay, we may be able to detect evidence of tidal or magnetic SPI (see, e.g., Shkolnik et al. 2009). As the star expands, those interactions would strengthen, which should lead to more rapid orbital decay. While typically tidal interaction leading to circularization and orbital decay has been modeled to depend only on tides in the planet, Jackson et al. (2008) show that tides in the star (which are strongly dependent on the stellar radius) can also influence orbital evolution. This provides a tidal mechanism for more distant planets to experience enhanced orbital evolution as the star expands. Recent simulations by Strugarek et al. (2014) further demonstrate that in some scenarios (especially with strong stellar magnetic fields and slow rotation), magnetic SPI can be as important to orbital migration as tidal SPI and can lead to rapid orbital decay. Kepler-432 does rotate slowly, and the results of a two-decade survey by Konstantinova-Antova et al. (2013) reveal that giants can exhibit field strengths up to $\sim 100$ G. Encouraged by these findings, we search for evidence of SPI in the Kepler-432 system in Section 9.5.

The hypothetical scenario in which planets inside $\sim 0.5 \mathrm{AU}$ around massive and intermediate-mass stars ultimately get destroyed may also help explain the properties of the observed distribution of planets orbiting evolved stars. If planets form on circular orbits, then in order to excite large eccentricities, a planet must experience dynamical interaction with another planet (e.g., Rasio \& Ford 1996; Juric \& Tremaine 2008) or another star (e.g., Fabrycky \& Tremaine 2007; Naoz et al. 2012). More massive stars (like many of those that have become giants) are more likely than lower mass, Sun-like stars to form giant planets, and they are also more likely to have binary companions (e.g., de Rosa et al. 2014; Raghavan et al. 2010), so one might expect the typical planet orbiting a giant star to be more eccentric, not less. However, the most eccentric planets around evolved stars would have pericenter distances near or inside the critical separation at which planets get destroyed. This could lead either to destruction by the same mechanisms as the close-in planets or to partial orbital decay and circularization. The consequence of either process would be more circular orbits on average and an overabundance of planets at separations near the critical separation (0.5 AU $\lesssim a \lesssim 1 \mathrm{AU})$, which is indeed observed. For similar arguments and additional discussion, see Jones et al. (2014). Counterarguments articulated by those authors include that massive subgiants do not seem to host many planets inside $1 \mathrm{AU}$ either, but subgiants are not large enough to have swallowed them yet. This might point toward a primordial difference in the planetary period distribution between Sun-like and more massive stars, rather than a difference that evolves over time. 


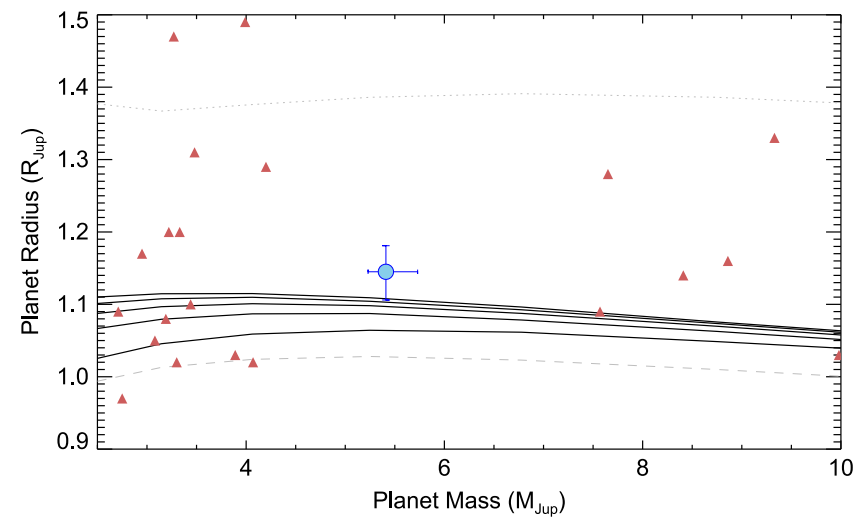

Figure 14. Masses and radii of known giant planets (red triangles) and Kepler$432 \mathrm{~b}$ (blue circle). Overplotted as solid black lines are a series of planetary models (Fortney et al. 2007) with age $3.5 \mathrm{Gyr}$, an appropriate semi-major axis, and core masses $0,10,25,50$, and $100 M_{\oplus}$ (from top to bottom). Also plotted are models for young (100 Myr), hot (0.02 AU), coreless planets (dotted gray line); and old $(10 \mathrm{Gyr})$, cool (1 AU) planets with massive $\left(100 M_{\oplus}\right)$ cores (dashed gray line). These roughly illustrate the range of sizes possible for a given mass.

\subsection{Implications for Giant Planet Formation and Migration}

Assuming that giant planets form beyond the ice line (located at $\sim 4.9 \mathrm{AU}$ for a star with $M \sim 1.4 M_{\odot}$, using the approximation of Ida \& Lin 2005), both Kepler-432b and c have experienced significant inward migration. At first glance, their large orbital eccentricities would suggest that they have experienced gravitational interactions, perhaps during one or more planet-planet scattering events that brought them to their current orbital distances, or through the influence of an outer companion (like that detected in our AO images). However, the apparent alignment between the stellar spin axis and the orbit of the inner planet presents a puzzle; if the (initially circular, coplanar) planets migrated via multi-body interactions, we would expect both the eccentricities and the inclinations to grow. It is possible, of course, that by chance the inner planet remained relatively well aligned after scattering, or that the planets experienced coplanar, higheccentricity migration, which has recently been suggested as a mechanism for producing hot Jupiters (Petrovich 2014). Measuring the mutual inclination between the planets could lend credence to one of these scenarios. Unfortunately, because we are unable to constrain the mutual inclination of the planets, we cannot say whether they are mutually well aligned (which would argue against planet-planet scattering) or not.

At this point, it is prudent to reiterate that the stellar spin and orbital angular momenta are measured to be nearly aligned only as projected along the line of sight. The true obliquity is unknown and may be significantly non-zero. In Figure 16, we illustrate this by simulating the underlying obliquity distribution that corresponds to an example $i_{\star}$ distribution with a mean of $83^{\circ}$, similar to that measured from asteroseismology. To generate this obliquity distribution, we draw from $i_{\star}$ and a random uniform azimuthal angle, $\theta$, and calculate the true obliquity, $\psi$. As is apparent from the $\psi$ distribution, while it is likely that the true obliquity is low, there is a tail of highly misaligned systems (representing those with spin axes lying nearly in the plane of the sky but not coincident with the orbital

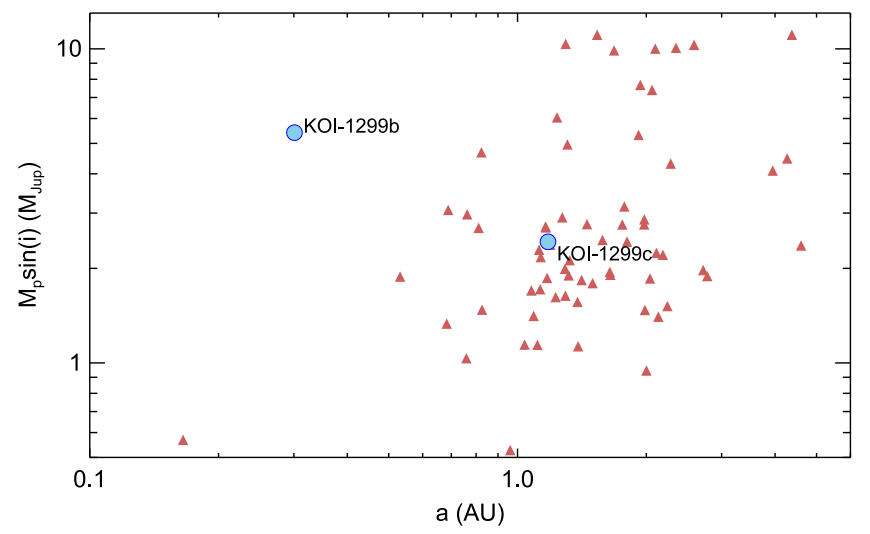

Figure 15. All planets with minimum masses $M_{\mathrm{p}} \sin i>0.5 M_{\mathrm{Jup}}$ orbiting giant stars $\left(\log g_{\star}<3.9\right)$, plotted as red triangles. Kepler $-432 \mathrm{~b}$ and $\mathrm{c}$ are labeled and plotted as large blue circles.

angular momentum) that could produce the stellar inclination we observe. Ideally, we would also measure the sky-projected spin-orbit angle, $\lambda$, in order to detect any such conspiring misalignment and calculate the true obliquity. However, there is not an obvious way to do this: the slow rotation, long period, and lack of star spots render current techniques to measure the sky-projected obliquity ineffective. We are left with an inner planet that is likely, but not certainly, aligned with the stellar spin axis, and an unknown inclination of the outer orbital plane.

The likely alignment of the inner planet seems at odds with the large orbital eccentricities and the assumption that multibody interactions are responsible for their migration, but we have thus far neglected interaction between the planets and the host star. In their investigation of hot Jupiter obliquities, Winn et al. (2010) suggested that well-aligned hot Jupiters may have been misaligned previously, and that subsequent tidal interaction may have realigned the stellar rotation with the planetary orbit, perhaps influencing the convective envelope independently of the interior. This idea was furthered by Albrecht et al. (2012), who found that systems with short tidal-dissipation timescales are likely to be aligned, while those with long timescales are found with a wide range of obliquities. In the following section, we explore the possibility that Kepler- $432 \mathrm{~b}$ has undergone a similar evolution, obtaining an inclined orbit through its inward migration, but subsequently realigning the stellar spin.

\subsection{Evidence for Spin-Orbit Evolution}

During the main-sequence lifetime of Kepler-432, the current position of the planets would be too far from the star to raise any significant tides, but the star is now several times its original size, such that at periastron, the inner planet passes within 7.7 stellar radii of the star. The unusually large planetary mass also helps strengthen tidal interactions. Following Albrecht et al. (2012) (who in turn used the formulae of Zahn 1977), for Kepler-432 we calculate the tidal timescale for a star with a convective envelope:

$$
\tau_{\mathrm{CE}}=\left(10^{10} \mathrm{yr}\right) q^{-2}\left(\frac{a / R_{\star}}{40}\right)^{6} .
$$




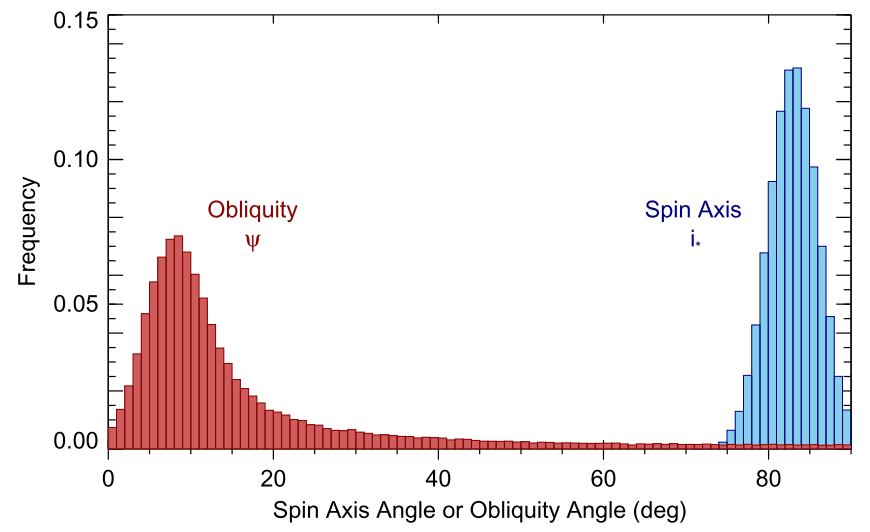

Figure 16. Underlying distribution of true obliquities, $\psi$, corresponding to a hypothetical measured stellar inclination, $i_{\star}$. A system with edge-on rotation has $i_{\star}=90^{\circ}$, and $\psi=0^{\circ}$ indicates perfect alignment between stellar spin and planetary orbital angular momentum. The long tail of high obliquities illustrates that even with a nearly edge-on stellar spin, a transiting planet may still be misaligned.

For a radiative atmosphere, as Kepler-432 would have had on the main sequence, the appropriate timescale would be

$$
\tau_{\mathrm{RA}}=\left(1.25 \times 10^{9} \mathrm{yr}\right) q^{-2}(1+q)^{-5 / 6}\left(\frac{a / R_{\star}}{6}\right)^{17 / 2}
$$

We repeat the words of caution from Albrecht et al. (2012): these equations are calibrated to star-star interactions rather than star-planet interactions, and are timescales for spin-orbit synchronization, not realignment. As such, the timescales are assumed to be valid only as a relative metric. The authors arbitrarily divided the resulting values by $5 \times 10^{9}$ to express this point, and to set the values on a convenient scale. In Figure 17, we have reproduced a version of Figure 24 of Albrecht et al. (2012), showing the relative tidal dissipation timescales for all hot Jupiters with measured spin-orbit angles. We have excluded the same systems they did and added nine recent projected obliquity measurements, including that of Kepler-432b. Their result still holds true: systems with short tidal dissipation timescales are well aligned, while those with long tidal timescales display a wide range of obliquities. We also note that recent work by Valsecchi \& Rasio (2014), which includes a more detailed treatment of the convection and stellar evolution of each star, similarly concludes that the observed obliquity distribution can be explained by tidal evolution. While the angle measured for Kepler-432b is the line-of-sight projection rather than the sky-plane projection, and the efficiency of realignment may be different for evolved stars given their different internal structure, Kepler-432b does have a short tidal timescale, and it sits in a region with hot Jupiter systems that are mostly well aligned. We interpret this result as evidence that the spin axis of Kepler-432 has been realigned to the orbit of the inner planet by the same mechanism responsible for hot Jupiter realignment.

Alternative mechanisms have been proposed to explain the alignment for hot Jupiters orbiting cool stars. One such alternative, suggested by Dawson (2014), is that the orbital alignment trend with stellar temperature is due to strong versus weak magnetic breaking (for stars below and above the Kraft break), rather than tidal dissipation efficiency as in Albrecht et al. (2012). This has the attractive quality of being part of a theory that is also able to predict the observed mass cut-off for

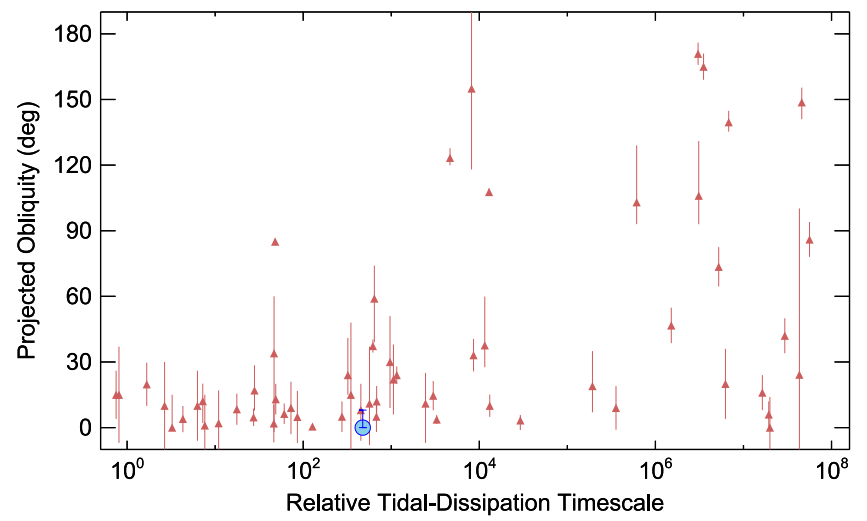

Figure 17. Adapted version of Figure 24 from Albrecht et al. (2012). We plot the projected obliquity of giant planets versus the relative tidal timescale. We include planets from Albrecht et al. (2012), as well as eight more recent spinorbit measurements from the literature (red triangles) and Kepler-432b (large blue circle). Because it sits in a region with well-aligned hot Jupiters, we posit that Kepler-432b has also realigned the spin of its host star.

retrograde planets and the trend between planet mass and host star rotation periods. In the Dawson (2014) framework, one may still conclude that Kepler-432 would realign during its red giant phase, but this depends in part on the unknown magnetic properties of the star: the Konstantinova-Antova et al. (2013) finding of strong magnetic fields in some giants may indeed indicate that Kepler-432 possesses a short enough magnetic braking timescale to allow for realignment in this framework, too.

In the event that the envelope and the core have different spin axis inclinations, this might manifest itself in the asteroseismic modes. Because g-mode-dominated $\ell=1$ modes are most sensitive to core rotation while p-mode-dominated mixed modes are most sensitive to the surface, one might obtain different inclination measurements from the different modes. However, our analysis of individual $\ell=1$ modes in Section 5.3 showed they were consistent with $\sim 90^{\circ}$ inclination for both core and envelope. This would argue against a realigned envelope for Kepler-432, but a modest misalignment might be undetectable given the degraded precision when fitting modes independently. Furthermore, as shown for the obliquity and spin axis in Figure 16, even if the two spin axes both lie in the sky plane, there is a chance they could be significantly misaligned. Regardless of its efficacy in the case of Kepler-432, asteroseismology may be able to detect a significant misalignment between core and envelope in other systems hosting giant planets with short tidal timescales. If detected, this would be important evidence in the interpretation of giant planet migration, and we suggest this be explored for other systems.

While the evidence presented thus far for realignment of the Kepler-432 system is circumstantial, there may be additional evidence to support the scenario. If tidal forces are strong enough to realign the spin axis of the host star, they may also excite activity on the stellar surface. SPI need not be limited to tidal interaction, though. At closest approach, the planet is only 7.7 stellar radii from the star, and thus magnetic interaction may also be possible, since the planet passes inside the Alfvén radius $\left(\sim 10 R_{\star}\right)$. We return to the light-curve to look for signs of these interactions. When folded on the orbital period, an interesting feature emerges (see Figure 18). Just after transit, we detect a brightening of $\sim 0.05$ mmag that maintains a 


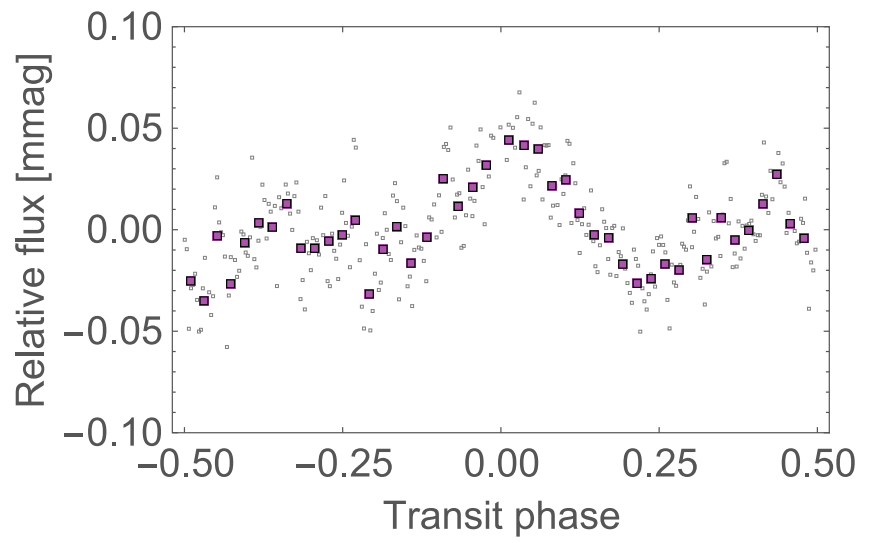

Figure 18. Kepler light-curve, binned and folded to the period of the transiting planet, with the transits removed. Given that periastron occurs just before transit and the stellar rotation period is longer than the orbital period, the brightening just after transit (orbital phase 0) is what would be expected for a bright spot that is tidally excited during the periastron passage of the planet.

coherent shape and phase throughout the mission. The simplest explanation for a light-curve feature with the period of the planet is a phenomenon associated with the planet. We suggest that SPI excites a bright spot on the stellar photosphere each orbit during periastron passage. Periastron occurs 1.24 days before transit, and because the rotation period is longer than the orbital period, the spot should rotate onto the meridian and reach maximum apparent brightness slightly after transit, as is observed.

One might argue that if the planet has realigned the stellar spin, it may have also synchronized it to the orbital periodthis would cause any long-lived bright spot to create a peak in the light-curve when folded to the orbital period. However, to obtain the observed phasing, the spot would have to conveniently occur at the longitude that coincides with the close approach of the planet. There is also no reason to expect a long-lived bright spot on the star, irrespective of its phasing. Furthermore, there exists evidence that the spin is not synchronized to the orbital period anyway. We have measured $R_{\star}, i_{\star}$, and $v \sin i_{\star}$, so we can estimate the rotation period to be $P_{\text {rot }} \sim 77 \pm 14$ days, which is inconsistent with the orbital period $\left(P_{\text {orb }}=52.5\right.$ days $)$. Thus, a long-lived rotating star spot should not produce a coherent photometric signal when folded to the orbital period. (Similarly, we must conclude that if $P_{\text {rot }} \neq P_{\text {orb }}$, any planet-induced spot must decay in brightness relatively quickly, or the brightening feature would appear at different phases in each orbit, washing out the coherent signal that we observe.) We do caution that our $v \sin i_{\star}$ measurement for this slowly rotating giant could be biased, for example, due to the unknown macroturbulent velocity of Kepler-432. However, even if our measurement of the rotational velocity is incorrect and the rotation period is synchronized to the orbit, we believe a bright star spot phased with the periastron passage of the planet would more easily be attributed to SPI than coincidence.

One additional worry would be that granulation could produce correlated noise that happens to fold coherently on the orbital period. We find this to be an unlikely scenario given the periodogram of the Kepler data (see Figure 19). If granulation produced light-curve amplitudes similar to that observed, we would expect several periods to show similar power simply by chance. Instead, we observe the peak power at the second

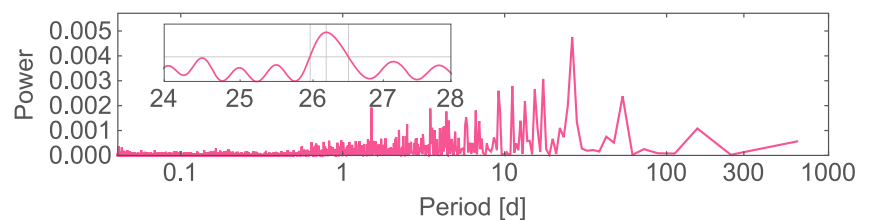

Figure 19. Periodogram of the Kepler light-curve, after transits were removed. The maximum power occurs at a period of $\sim 26.2$ days, or half of the orbital period of the inner planet.

harmonic of the orbital period ( $\sim 26.2$ days $)$, and very little power at other periods. It is not particularly surprising that the second harmonic, rather than the first, is the strongest peak, especially given that there is some additional structure in the light-curve at phases other than around transit.

The observant reader might notice a possible brightening of lower amplitude at phase $\sim 0.4$ of the folded light-curve. We are not confident that this is real, but if it is, it holds a wealth of information. As mentioned above, any excited spot must decay in brightness quickly, or much of the star would soon be covered in such spots, obfuscating the coherent signal that we observe. If, however, a spot excited during periastron lasted only slightly more than one rotation period, we would expect to see a second peak in the folded light-curve, with an amplitude dependent on the spot lifetime and phase dependent on the rotation period. For Kepler-432, the second peak $\sim 1.4$ orbits after the first would imply a rotation period of $\sim 1.4 \times 52.5$ days $\approx 73.5$ days, perfectly consistent with our independently estimated rotation period. The amplitude of the second peak is roughly half that of the first, so we could conclude that the characteristic lifetime (half-life) of such a spot is roughly the same as the rotation period, $\tau_{\text {spot }} \sim 73.5$ days. We reiterate that we are not confident that the data here are strong enough to make such conclusions; we merely note the consistent rotation period it would imply.

\section{SUMMARY}

We have presented herein the discovery of the Kepler-432 planetary system, consisting of two giant planets orbiting a red giant star, and a faint visual companion that is probably a physically bound $\mathrm{M}$ dwarf with an orbital separation of at least $750 \mathrm{AU}$. The inner planet $(P=52.5$ days $)$ transits the star, allowing a more detailed study of its properties.

An asteroseismic analysis of the host star allows precise measurements of the stellar mass, radius, age, and spin axis inclination. This in turn leads to precise planetary properties, especially for the transiting inner planet. $N$-body simulations have helped constrain the properties of the outer planet by invoking stability arguments to rule out a large fraction of orbital solutions.

Kepler-432b is among the most massive transiting planets orbiting any type of star. Furthermore, it is not highly irradiated like many of the more common short-period transiting planets, experiencing only $\sim 20 \%$ of the insolation that a 3-day hot Jupiter orbiting a Sun-like star would receive. This makes it an interesting benchmark for interior models and a good test of planetary inflation at moderate insolation. It appears to be slightly inflated compared to the models but is marginally consistent with having no core (or a small core) of heavy elements.

The eccentricities of both planets are high, which is suggestive of migration through multi-body interactions. 
Puzzlingly, the transiting planet is likely well aligned with the stellar spin, which is not an expected property of systems that have experienced such interactions. However, subsequent tidal or magnetic interaction with the host star may reconcile the two results. We find that because of the large stellar radius and planetary mass, the tidal dissipation timescale of the system is similar to that of hot Jupiters that are well aligned. Those planets are thought to be well aligned due to reorientation of the angular momenta after the initial inward migration; we conclude that the same process that realigns hot Jupiter systems may have also realigned this system, despite its long period.

Under the assumption that the star and planet are interacting strongly enough to realign the stellar spin, we searched for evidence of this interaction in the Kepler photometry. The star is photometrically quiet on long timescales, which allowed us to detect a low-amplitude brightening soon after periastron. We conclude that the most likely explanation is a bright spot excited (either tidally or magnetically) by the planet during its close approach to the stellar photosphere. This evidence for ongoing SPI further supports the obliquity realignment scenario.

Finally, we note that Kepler-432b is an outlier among giant planets orbiting giant stars. It is one of only three such planets orbiting within $0.5 \mathrm{AU}$ of its host star. Either it is intrinsically rare, or it is one of a more common class of planets orbiting interior to $1 \mathrm{AU}$ that get destroyed relatively quickly once the star reaches the red giant branch. Given that Kepler-432 has only recently started its ascent up the RGB — most red giants are in a more advanced evolutionary state-and there is already evidence for SPI, it is plausible that many more red giants initially hosted planets similar to Kepler-432b that have subsequently been engulfed. Further investigation of this group of planets will provide more clarity and ultimately have strong implications for giant planet formation around intermediateand high- mass stars, which are more difficult to study on the main sequence because of their high temperatures and rapid rotation.

We thank Russel White and an anonymous referee for valuable discussion and feedback. S.N.Q. is supported by the NSF Graduate Research Fellowship, Grant DGE-1051030. D.W.L. acknowledges partial support from NASA's Kepler mission under Cooperative Agreement NNX11AB99A with the Smithsonian Astrophysical Observatory. D.H. acknowledges support by the Australian Research Council's Discovery Projects funding scheme (project number DEI40101364) and support by NASA under Grant NNX14AB92G issued through the Kepler Participating Scientist Program. M.J.P. gratefully acknowledges the NASA Origins of Solar Systems Program grant NNX13A124G. Funding for the Stellar Astrophysics Centre is provided by The Danish National Research Foundation (Grant DNRF106). The research is supported by the ASTERISK project (ASTERoseismic Investigations with SONG and Kepler) funded by the European Research Council (Grant agreement no.: 267864). The research leading to the presented results has also received funding from the European Research Council under the European Community's Seventh Framework Programme (FP7/2007-2013)/ERC grant agreement no 338251 (StellarAges). This publication makes use of data products from the Two Micron All Sky Survey, which is a joint project of the University of Massachusetts and the Infrared Processing and Analysis Center/California Institute of
Technology, funded by the National Aeronautics and Space Administration and the National Science Foundation. This research has also made use of the APASS database, located at the AAVSO Web site. Funding for APASS has been provided by the Robert Martin Ayers Sciences Fund.

Facilities: Kepler, FLWO:1.5 m (TRES), NOT (FIES), Keck:II (NIRC2), WIYN (DSSI)

\section{REFERENCES}

Aerts, C., Christensen-Dalsgaard, J., \& Kurtz, D. W. 2010, Asteroseismology (Berlin: Springer)

Aizenman, M., Smeyers, P., \& Weigert, A. 1977, A\&A, 58, 41

Albrecht, S., Winn, J. N., Johnson, J. A., et al. 2012, ApJ, 757, 18

Appourchaux, T., Chaplin, W. J., García, R. A., et al. 2012, A\&A, 543, 54

Barclay, T., Endl, M., Huber, D., et al. 2015, ApJ, 800, 46

Barnes, J. W., Linscott, E., \& Shporer, A. 2011, ApJS, 197, 10

Basu, S., Chaplin, W. J., \& Elsworth, Y. 2010, ApJ, 710, 1596

Batalha, N. M., Rowe, J. F., Bryson, S. T., et al. 2013, ApJS, 204, 24

Beck, P. G., Hambleton, K., Vos, J., et al. 2014, A\&A, 564, 36

Beck, P. G., Montalban, J., Kallinger, T., et al. 2012, Natur, 481, 55

Bedding, T. R., Mosser, B., Huber, D., et al. 2011, Natur, 471, 608

Belkacem, K., Goupil, M. J., Dupret, M. A., et al. 2011, A\&A, 530, 142

Benomar, O., Masuda, K., Shibahashi, H., \& Suto, Y. 2014, PASJ, 66, 94

Berta, Z. K., Charbonneau, D., Désert, J.-M., et al. 2012, ApJ, 747, 35

Borucki, W. J., Koch, D., Basri, G., et al. 2010, Sci, 327, 977

Bowler, B. P., Johnson, J. A., Marcy, G. W., Henry, G. W., et al. 2010, ApJ, 709, 396

Brandão, I. M., Doğan, G., Christensen-Dalsgaard, J., et al. 2011, A\&A 527,37

Bressan, A., Marigo, P., Girardi, L., et al. 2012, MNRAS, 427, 127

Brown, T. M., Latham, D. W., Everett, M. E., \& Esquerdo, G. A. 2011, AJ, 142,112

Buchhave, L. A., Bakos, G. Á, Hartman, J. D., et al. 2010, ApJ, 720, 1118

Buchhave, L. A., Bizzarro, M., Latham, D. W., et al. 2014, Natur, 509, 593

Buchhave, L. A., Latham, D. W., Johansen, A., et al. 2012, Natur, 486, 375

Buchner, J., Georgakakis, A., Nandra, K., et al. 2014, A\&A, 564, 125

Casella, G., \& George, E. I. 1992, Am. Stat., 46, 3

Chaplin, W. J., Basu, S., Huber, D., et al. 2014, ApJS, 210, 1

Chaplin, W. J., Sanchis-Ojeda, R., Campante, T. L., et al. 2013, ApJ, 766, 101

Charbonneau, D., Brown, T. M., Noyes, R. W., \& Gilliland, R. L. 2002, ApJ, 568,377

Chen, Y., Girardi, L., Bressan, A., et al. 2014, MNRAS, 444, 2525

Christensen-Dalsgaard, J. 2008a, Ap\&SS, 316, 13

Christensen-Dalsgaard, J. 2008b, Ap\&SS, 316, 113

Ciceri, S., Lillo-Box, J., Southworth, J., et al. 2015, A\&A, 573, L5

Collier Cameron, A., Bruce, V. A., Miller, G. R. M., et al. 2010, MNRAS, 403, 151

Dawson, R. I. 2014, ApJL, 790, 31

Dawson, R. I., \& Johnson, J. A. 2012, ApJ, 756, 122

de Rosa, R. J., Patience, J., Wilson, P. A., et al. 2014, MNRAS, 437, 1216

Désert, J.-M., Charbonneau, D., Demory, B.-O., Ballard, S., et al. 2011, ApJS, 197, 14

Djupvik, A. A., \& Andersen, J. 2010, in Highlights of Spanish Astrophysics, ed. J. M. Diego et al. (5th ed., Berlin: Springer)

Döllinger, M. P., Hatzes, A. P., Pasquini, L., Guenther, E. W., \& Hartmann, M. 2009, A\&A, 505, 1311

Fabrycky, D., \& Tremaine, S. 2007, ApJ, 669, 1298

Feroz, F., Hobson, M. P., \& Bridges, M. 2009, MNRAS, 398, 1601

Feroz, F., Hobson, M. P., Cameron, E., \& Pettitt, A. N. 2013, arXiv:1306.2144 Fortney, J. J., Marley, M. S., \& Barnes, J. W. 2007, ApJ, 659, 1661

Frandsen, S., \& Lindberg, B. 1999, in Astrophysics with the NOT, ed. H. Karttunen \& V. Piirola (Univ. Turku: Piikkio), 71

Fúrész, G. 2008, Ph.D. thesis, Univ. Szeged, Hungary

Gai, N., Basu, S., Chaplin, W. J., \& Elsworth, Y. 2011, ApJ, 730, 63

Girardi, L., Groenewegen, M. A. T., Hatziminaoglou, E., \& da Costa, L. 2005, A\&A, 436, 895

Gizon, L., \& Solanki, S. K. 2003, ApJ, 589, 1009

Goldreich, P., \& Tremaine, S. 1980, ApJ, 241, 425

Handberg, R., \& Campante, T. L. 2011, A\&A, 527, 56

Handberg, R., \& Lund, M. N. 2014, MNRAS, 445, 2698

Hastings, W. H. 1970, Biometrika, 57, 97

Horch, E. P., Falta, D., Anderson, L. M., et al. 2010, AJ, 139, 205 
Houdek, G., Balmforth, N. J., Christensen-Dalsgaard, J., \& Gough, D. O. 1999, A\&A, 351, 582

Howell, S. B., Everett, M. E., Sherry, W., et al. 2011, AJ, 142, 19

Huber, D., Carter, J. A., Barbieri, M., et al. 2013b, Sci, 342, 331

Huber, D., Chaplin, W. J., Christensen-Dalsgaard, J., et al. 2013a, ApJ, 767, 127

Ida, S., \& Lin, D. N. C. 2005, ApJ, 626, 1045

Jackson, B., Greenberg, R., \& Barnes, R. 2008, ApJ, 678, 1396

Jiang, C., Jiang, B. W., Christensen-Dalsgaard, J., et al. 2011, ApJ, 742, 120

Johnson, J. A., Aller, K. M., Howard, A. W., \& Crepp, J. R. 2010, PASP 122,905

Johnson, J. A., Clanton, C., Howard, A. W., Bowler, B. P., et al. 2011, ApJS, 197, 26

Johnson, J. A., Fischer, D. A., Marcy, G. W., Wright, J. T., et al. 2007, ApJ, 665,785

Johnson, M. C., Cochran, W. D., Albrecht, S., et al. 2014, ApJ, 790, 30

Jones, M. I., Jenkins, J. S., Bluhm, P., Rojo, P., \& Melo, C. H. F. 2014, A\&A, 566,113

Juric, M., \& Tremaine, S. 2008, ApJ, 686, 603

Kallinger, T., Weiss, W. W., Barban, C., et al. 2010, A\&A, 509, 77

Kipping, D. M. 2008, MNRAS, 389, 1383

Kipping, D. M. 2010, MNRAS, 408, 1758

Kipping, D. M. 2013a, MNRAS, 435, 2152

Kipping, D. M. 2013b, MNRAS, 434, 51

Kipping, D. M. 2014a, MNRAS, 440, 2164

Kipping, D. M. 2014b, MNRAS, 444, 2263

Kipping, D. M., Dunn, W. R., Jasinski, J. M., \& Manthri, V. P. 2012, MNRAS, 421, 1166

Kipping, D. M., Hartman, J., Buchhave, L. A., et al. 2013, ApJ, 770, 101

Kjeldsen, H., \& Bedding, T. R. 1995, A\&A, 293, 87

Kjeldsen, H., Bedding, T. R., \& Christensen-Dalsgaard, J. 2008, ApJL, 683,175

Knutson, H. A., Charbonneau, D., Allen, L. E., et al. 2008, ApJ, 673, 526

Koch, D. G., Borucki, W. J., Basri, G., et al. 2010, ApJL, 713, 79

Konstantinova-Antova, R., Aurére, M., Charbonnel, C., Wade, G., et al. 2013, BlgAJ, 19, 14

Kraft, R. P. 1967, ApJ, 150, 551

Kurucz, R. L. 1992, IAUS, 149, 225

Lillo-Box, J., Barrado, D., Henning, T., et al. 2014a, A\&A, 568, L1

Lillo-Box, J., Barrado, D., Moya, A., et al. 2014b, A\&A, 562, 109

Lin, D. N. C., \& Papaloizou, J. 1986, ApJ, 309, 846

Lomb, N. R. 1976, Ap\&SS, 39, 447

Lovis, C., \& Mayor, M. 2007, A\&A, 472, 657

Lund, M. N., Lundkvist, M., Silva Aguirre, V., et al. 2014, A\&A, 570, 54

Mandel, K., \& Agol, E. 2002, ApJL, 580, 171

Mandushev, G., Torres, G., Latham, D. W., et al. 2005, ApJ, 621, 1061

Mathur, S., Hekker, S., Trampedach, R., et al. 2011, ApJ, 741, 119

McLaughlin, D. B. 1924, ApJ, 60, 22

Metcalfe, T. S., Monteiro, M. J. P. F. G., Thompson, M. J., et al. 2010, ApJ, 723,1583

Metropolis, N., Rosenbluth, A. W., Rosenbluth, M. N., et al. 1953, JhPh, 21,1087

Mosser, B., Goupil, M. J., Belkacem, K., et al. 2012a, A\&A, 540, 143
Mosser, B., Goupil, M. J., Belkacem, K., et al. 2012b, A\&A, 548, 10

Naoz, S., Farr, W. M., \& Rasio, F. A. 2012, ApJL, 754, 36

Nidever, D. L., Marcy, G. W., Butler, P. R., et al. 2002, ApJS, 141, 503

Nutzman, P. A., Fabrycky, D. C., \& Fortney, J. J. 2011, ApJL, 740, 10

Ortiz, M., Gandolfi, D., Reffert, S., et al. 2015, A\&A, 573, L6

Paxton, B., Cantiello, M., Arras, P., et al. 2013, ApJS, 208, 4

Payne, M. J., Deck, K. M., Holman, M. J., \& Perets, H. B. 2013, ApJL, 775, L44

Petrovich, C. 2014, ApJ submitted (arXiv:1409.8296)

Poppenhaeger, K., Schmitt, J. H. M. M., \& Wolk, S. J. 2013, ApJ, 773, 62

Queloz, D., Henry, G. W., Sivan, J. P., et al. 2001, A\&A, 379, 279

Quinn, S. N., White, R. J., Latham, D. W., et al. 2014, ApJ, 787, 27

Raghavan, D., McAlister, H. A., Henry, T. J., et al. 2010, ApJS, 190, 1

Rasio, F. A., \& Ford, E. B. 1996, Sci, 274, 954

Rauch, K. P., \& Holman, M. 1999, AJ, 117, 1087

Rossiter, R. A. 1924, ApJ, 60, 15

Saar, S. H., \& Donahue, R. A. 1997, ApJ, 485, 319

Sanchis-Ojeda, R., Winn, J. N., Holman, M. J., Carter, J. A., et al. 2011, ApJ, 733,127

Sato, B., Izumiura, H., Toyota, E., Kambe, E., et al. 2008, PASJ, 60, 539

Scargle, J. D. 1982, ApJ, 263, 835

Schlafly, E. F., \& Finkbeiner, D. P. 2011, ApJ, 737, 103

Schlegel, D. J., Finkbeiner, D. P., \& Davis, M. 1998, ApJ, 500, 525

Shkolnik, E., Aigrain, S., Cranmer, S., et al. 2009, in AIP Conf. Proc. 1094, Cool Stars, Stellar Systems, and the Sun, ed. E. Stempels (Melville, NY: AIP), 275

Silva Aguirre, V., Casagrande, L., Basu, S., et al. 2012, ApJ, 757, 99

Silva Aguirre, V., Ruchti, G. R., Hekker, S., et al. 2014, ApJ, 784, 16

Skrutskie, M. F., Cutri, R. M., Stiening, R., et al. 2006, AJ, 131, 1163

Sliski, D. H., \& Kipping, D. M. 2014, ApJ, 788, 148

Steffen, J. H., Ragozzine, D., Fabrycky, D. C., et al. 2012, PNAS, 109, 7982

Stello, D., Bruntt, H., Preston, H., \& Buzasi, D. 2008, ApJ, 674, 53

Stello, D., Chaplin, W. J., Bruntt, H., et al. 2009, ApJ, 700, 1589

Stello, D., Huber, D., Bedding, T. R., et al. 2013, ApJ, 765, 41

Strugarek, A., Brun, A. S., Matt, S. P., \& Réville, V. 2014, ApJ, 795, 86

Tingley, B., Bonomo, A. S., \& Deeg, H. J. 2011, ApJ, 726, 112

Torres, G., Fressin, F., Batalha, N. M., et al. 2011, ApJ, 727, 24

Torres, G., Fischer, D. A., Sozzetti, A., et al. 2012, ApJ, 757, 161

Torres, G., Konacki, M., Sasselov, D. D., \& Jha, S. 2005, ApJ, 619, 558

Townsend, R. H. D., \& Teitler, S. A. 2013, MNRAS, 435, 3406

Valsecchi, F., \& Rasio, F. A. 2014, ApJ, 786, 102

van Cleve, J. 2008, Kepler, Instrument Handbook (available at http://archive stsci.edu/kepler/)

Van Eylen, V., Lund, M. N., Silva Aguirre, V., et al. 2014, ApJ, 782, 14

Weiss, A., \& Schlattl, H. 2008, Ap\&SS, 316, 99

Winn, J. N., Fabrycky, D., Albrecht, S., \& Johnson, J. A. 2010, ApJL, 718,145

Wu, Y., \& Lithwick, Y. 2011, ApJ, 735, 109

Wu, Y., \& Murray, N. 2003, ApJ, 589, 605

Zacharias, N., Finch, C. T., Girard, T. M., et al. 2013, AJ, 145, 44

Zahn, J.-P. 1977, A\&A, 57, 383 